$\mathrm{UW} / \mathrm{PT} 98-11$

\title{
Longitudinal subtleties in diffusive Langevin equations for non-Abelian plasmas
}

\author{
Peter Arnold \\ Department of Physics, University of Virginia, Charlottesville, VA 22901 \\ Dam T. Son \\ Center for Theoretical Physics, Department of Physics, \\ Massachusetts Institute of Technology, Cambridge, MA 02139 \\ Laurence G. Yaffe \\ Department of Physics, University of Washington, Seattle, Washington 98195
}

(January 1999)

\begin{abstract}
Bödeker has recently argued that non-perturbative processes in very high temperature non-Abelian plasmas (such as electroweak baryon number violation in the very hot early Universe) can be quantitatively described, to leading logarithmic accuracy, by a simple diffusive effective theory. Bödeker's effective theory is intended to describe the long-distance transverse electric and magnetic fields which are responsible for non-perturbative dynamics. His effective theory, however, also contains long-wavelength longitudinal electric fields. We discuss several subtleties in the treatment of longitudinal dynamics which were not closely examined in Bödeker's original treatment. Somewhat to our surprise, we find that within its domain of validity Bödeker's effective theory does correctly describe both longitudinal and transverse fluctuations. We also show that, as far as the transverse dynamics of interest is concerned, Bödeker's effective theory could be replaced by a transverse-only theory that removes the longitudinal dynamics altogether. In the process, we discuss several interesting aspects of stochastic field theories.
\end{abstract}

\section{INTRODUCTION}

Non-perturbative processes in a hot non-Abelian plasma at or near equilibrium are associated with slow evolution of magnetic gauge fields. The characteristic spatial scale $R$ of

\footnotetext{
${ }^{1}$ This is explicitly argued in ref. [1], but this fact is also implicit in earlier analysis of specific thermal effects such as plasmon damping rates of fast-moving particles [2] and the color conductivity [5].
} 
non-perturbative gauge field fluctuations and the associated time scale $t$ for their evolution are of order

$$
R \sim \frac{1}{g^{2} T}, \quad t \sim \frac{1}{g^{4} T \ln (1 / g)}
$$

for small coupling. Alternatively, the characteristic spatial momentum $k$ and frequency $\omega$ are

$$
k \sim g^{2} T, \quad \omega \sim g^{4} T \ln (1 / g) .
$$

For a review, see the introduction of our earlier paper [6]. The logarithm appearing in the time scale is a recent and interesting result of Bödeker [7], whose physical interpretation we discuss in ref. [6].

Throughout this discussion, "hot" means that the temperature is large enough that the running coupling $\alpha(T)$ is small, that chemical potentials are ignorable, and that there is no spontaneous symmetry breaking. Examples of non-perturbative processes include chirality violation in hot QCD, and baryon number violation in hot electroweak theory (in its hightemperature symmetric phase).

Bödeker [7] has proposed an effective theory appropriate for the scales (1.1) above. His effective theory is a classical field theory that involves only gauge fields with dynamics governed by the diffusive Langevin equation

$$
\sigma \mathbf{E}=\mathbf{D} \times \mathbf{B}-\boldsymbol{\zeta}
$$

Here, $\mathbf{D}$ is the covariant derivative acting in the adjoint representation. In Bödeker's proposal, $\boldsymbol{\zeta}$ is a Gaussian white noise random force, normalized ast

$$
\left\langle\zeta_{i}^{a}(t, \mathbf{x}) \zeta_{j}^{b}\left(t^{\prime}, \mathbf{x}^{\prime}\right)\right\rangle=2 \sigma g^{2} T \delta^{a b} \delta_{i j} \delta\left(t-t^{\prime}\right) \delta^{(3)}\left(\mathbf{x}-\mathbf{x}^{\prime}\right),
$$

where $i, j$ and $a, b$ are spatial vector and adjoint color indices, respectively. This effective theory is supposed to give a quantitative description of non-perturbative physics in the hot plasma to leading order in the logarithm of the coupling. In other words, corrections to this effective theory are suppressed only by powers of $1 / \ln (1 / g)$. In ref. [6], we showed that $\sigma$ can be interpreted as the color conductivity of the plasma, which is given by [0, ,7, [6]

$$
\sigma \approx \frac{m_{\mathrm{pl}}^{2}}{\gamma_{\mathrm{g}}},
$$

\footnotetext{
${ }^{2}$ We will scale our gauge fields by a factor of $g$, so covariant derivatives contain no explicit couplings while the action (or energy) has an overall factor of $1 / g^{2}$. In addition, we will take the gauge group generators, and also the gauge field $\mathbf{A} \equiv \mathbf{A}^{a} T^{a}$, to be anti-Hermitian. Hence, the covariant derivative is simply $\mathbf{D}=\boldsymbol{\nabla}+\mathbf{A}$, and for the adjoint representation $\left(T^{a}\right)_{b c}=f_{b a c}$ with the structure constants $f_{a b c}$ real and totally anti-symmetric.

3 We are using "color" as a descriptive name for some non-Abelian gauge field. It should be emphasized that all discussion of "color" is applicable to the dynamics of, in particular, the SU(2) electroweak gauge field.
} 
where $m_{\mathrm{pl}}$ is the plasma frequency and

$$
\gamma_{\mathrm{g}} \approx \alpha C_{\mathrm{A}} T \ln (1 / g)
$$

is the damping rate for hard thermal gauge bosons 20 . The $\approx$ sign indicates equality at leading logarithmic order. [That is, we are not distinguishing $\ln (2 / g)$ from $\ln (1 / g)$ in Eq. 1.5, but the coefficient of the logarithm is correct.] The plasma frequency $m_{\mathrm{pl}}$ is well known at leading order in coupling and is of order $g T$.

Bödeker's effective theory is well suited to numerical simulation because it is classical, insensitive to ultraviolet cut-offs [6], and when cast into $A_{0}=0$ gauge generates a straightforward local equation of motion for the evolution of $\mathbf{A}$ :

$$
\sigma \frac{d}{d t} \mathbf{A}=-\mathbf{D} \times \mathbf{D} \times \mathbf{A}+\boldsymbol{\zeta} .
$$

A numerical investigation of Bödeker's effective theory and its implication for electroweak baryon number violation has been recently carried out by Moore [9].

Nevertheless, there is something peculiar about the effective theory (1.2). In a high temperature plasma, static electric fields (or, more generally, longitudinal fields) are Debye screened [10]. The screening distance is of order $1 / g T$, which is small compared to the spatial scale $R \sim 1 / g^{2} T$ of interest to us. More generally, the longitudinal modes of the gauge field are screened while, at low frequencies $\omega \ll k$, the transverse modes are not. Because of Debye screening, it is the transverse electric and magnetic fields which are relevant for producing non-perturbative fluctuations at the scales (1.1) quoted earlier. Longitudinal fields are irrelevant. Nonetheless, Bödeker's effective theory (1.2) does describe long-distance longitudinal fluctuations. The longitudinal fields are those pieces of $\mathbf{E}$ which contribute to $\mathbf{D} \cdot \mathbf{E}$ and which perturbatively correspond to polarizations parallel to the momentum $\mathbf{k}$. Dotting $\mathbf{D}$ into both sides of (1.2), one sees that

$$
\sigma \mathbf{D} \cdot \mathbf{E}=-\mathbf{D} \cdot \boldsymbol{\zeta}
$$

$\mathbf{D} \cdot \mathbf{E}$ is therefore not zero, and so the fields in Bödeker's effective theory (1.2) are not purely transverse. Our purpose in this paper will be to show two things: first, that Bödeker's original derivation of the long-distance longitudinal dynamics relied on a questionable approximation which ignored subtleties associated with longitudinal dynamics, but that the end result is nevertheless correct; and, secondly, that the longitudinal dynamics is irrelevant and may be removed altogether, if one is purely interested in describing physical, gauge-invariant quantities that depend only on the transverse fields (for example, the rate of anomalous charge violation). The last point would be trivial in an Abelian theory, because then (1.2) would be linear in the fields and could be projected into one equation involving only the transverse fields and another independent equation involving only the longitudinal fields. The point is much less trivial in a non-Abelian theory because of the non-linearity of (1.2).

\footnotetext{
${ }^{4}$ For hot electroweak theory with a single Higgs doublet, for instance, $m_{\mathrm{pl}}^{2}=\frac{1}{18}\left(5+2 n_{\mathrm{f}}\right) g^{2} T^{2}$ at leading order in $g$, where $n_{\mathrm{f}}=3$ is the number of fermion families, and the adjoint Casimir $C_{\mathrm{A}}=2$ in $(1.5)$. For QCD with $n$ flavors of quarks, $m_{\mathrm{pl}}^{2}=\frac{1}{3}\left(1+\frac{n}{6}\right) g^{2} T^{2}$ and $C_{\mathrm{A}}=3$, where $n$ is the number of relevant quark flavors (u, d, s, c, b, t).
} 


\section{REVIEW OF BÖDEKER'S DERIVATION}

\section{A. The effective Boltzmann-Vlasov equation}

We will refer to gauge fields associated with the scales of interest (1.1) as "soft" fields. In contrast, the dominant excitations in the hot plasma correspond to momenta of order $T$ and will be called "hard." On his way to deriving the effective theory (1.2) for the soft gauge fields, Bödeker [7] first derived an effective Boltzmann-Vlasov equation for the interaction of those fields with hard excitations:

$$
\begin{gathered}
\left(D_{t}+\mathbf{v} \cdot \mathbf{D}_{\mathbf{x}}\right) W-\mathbf{E} \cdot \mathbf{v}-\xi=-\delta C[W] \\
D_{\nu} F^{\mu \nu}=J^{\mu} \equiv m_{\mathrm{D}}^{2}\left\langle v^{\mu} W(\mathbf{v})\right\rangle_{\mathbf{v}}
\end{gathered}
$$

where

$$
\delta C[W](\mathbf{v}) \equiv \gamma_{\mathrm{g}}\left\langle\mathcal{I}\left(\mathbf{v}, \mathbf{v}^{\prime}\right) W\left(\mathbf{v}^{\prime}\right)\right\rangle_{\mathbf{v}^{\prime}}
$$

and

$$
\mathcal{I}\left(\mathbf{v}, \mathbf{v}^{\prime}\right) \equiv \delta^{(2)}\left(\mathbf{v}-\mathbf{v}^{\prime}\right)-\frac{4}{\pi} \frac{\left(\mathbf{v} \cdot \mathbf{v}^{\prime}\right)^{2}}{\sqrt{1-\left(\mathbf{v} \cdot \mathbf{v}^{\prime}\right)^{2}}}
$$

Here, $m_{\mathrm{D}}^{2} \equiv 3 m_{\mathrm{pl}}^{2}$ is the leading-order Debye mass (squared). The first equation (2.1a) is a linearized Boltzmann equation for the hard particles in the presence of a soft electromagnetic field, where $W(\mathbf{v}, \mathbf{x}, t)$ represents the color distribution of those particles and $\mathbf{v}$ is a unit vector representing the hard particles' velocities. . $\delta C$ represents a linearized collision term for $2 \rightarrow 2$ scattering that randomizes the color charges of the hard particles [6], and $\xi$ is a source of random thermal noise. The second equation (2.1b) is Maxwell's equation, where all the fields on the left-hand side are to be understood as soft fields, and the current on the right side is the soft-momentum component of the current created by hard excitations. This current is proportional to the density $W$ of hard particles and the velocities of those particles, where $v^{\mu}$ means $(1, \mathbf{v})$. In the explicit form (2.2) for the collision term, $\langle\cdots\rangle_{\mathbf{v}}$ denotes averaging over the direction of $\mathbf{v}$ and $\delta^{(2)}$ is a $\delta$ function defined on the unit twosphere with normalization

$$
\left\langle\delta^{(2)}\left(\mathbf{v}-\mathbf{v}^{\prime}\right) f\left(\mathbf{v}^{\prime}\right)\right\rangle_{\mathbf{v}^{\prime}}=f(\mathbf{v})
$$

See Refs. [7, 6] for the derivation of the explicit form (2.2b) of the linearized collision operator.

One may avoid worrying about the details of noise terms such as $\xi$ until one reaches the final effective equation (1.2), at which point it is possible to then argue how the noise must in

\footnotetext{
${ }^{5}$ Technically, $W$ is the adjoint representation piece of the density matrix describing the color charges of the hard excitations, summed over the various species of excitations and integrated over the energy of excitations (for a fixed direction of motion v). It is normalized in a way that simplifies the resulting equation. See ref. [6] for the explicit definition.
} 
fact appear [6]. However, since in this paper we will be discussing various subtleties, it will be useful to keep track of the noise explicitly at each step we consider. In particular, Bödeker [7] derived that the appropriate normalization of the noise in the effective Boltzmann-Vlasov equation (2.1) is related to the collision integral:

$$
\left\langle\xi^{a}(t, \mathbf{x}, \mathbf{v}) \xi^{b}\left(t^{\prime}, \mathbf{x}^{\prime}, \mathbf{v}^{\prime}\right)\right\rangle=\frac{2 g^{2} T}{3 \sigma} \mathcal{I}\left(\mathbf{v}, \mathbf{v}^{\prime}\right) \delta^{a b} \delta\left(t-t^{\prime}\right) \delta^{(3)}\left(\mathbf{x}-\mathbf{x}^{\prime}\right)
$$

We will not review any further the origin of the effective Boltzmann-Vlasov equations (2.1) and direct the reader instead to Bödeker's original work [7] and our alternative derivation [6]. It is in the step from these kinetic equations to Bödeker's final effective theory (1.2) that subtleties in the treatment of longitudinal physics creep in, and that is the focus of this paper.

\section{B. Solving for $W$}

Bödeker obtains his final effective theory (1.2), at leading-log order, from the effective Boltzmann-Vlasov equations (2.1) by arguing that the covariant derivative terms in the Boltzmann equation (2.1a) are together of order $g^{2} T W$ and so can be ignored compared to the collision term, which is of order $\gamma_{\mathrm{g}} W \sim\left(g^{2} T \ln g^{-1}\right) W$ and hence larger by a logarithm. There is an important subtlety to this approximation which will be examined in the next section. But accepting this argument at face value for now, if one drops the covariant derivative terms then the Boltzmann equation becomes simply

$$
\mathbf{E} \cdot \mathbf{v}+\xi \approx \delta C[W]
$$

Formally, the solution is

$$
W=(\delta C)^{-1}(\mathbf{E} \cdot \mathbf{v}+\xi)
$$

where $\delta C$ is to be understood here as an operator acting on the space of (adjointrepresentation) functions of a unit vector $\mathbf{v}$. This result for $W$ yields the spatial current appearing in 2.1b),

$$
\mathbf{J}=m_{\mathrm{D}}^{2}\left\langle\mathbf{v}(\delta C)^{-1}(\mathbf{E} \cdot \mathbf{v}+\xi)\right\rangle_{\mathbf{v}}
$$

Next note that $\delta C$ preserves the parity (in $\mathbf{v}$ ) of functions it acts on. In other words, $\delta C$ maps even (odd) functions of $\mathbf{v}$ into even (odd) functions of $\mathbf{v}$. (In contrast, the $\mathbf{v} \cdot \mathbf{D}_{\mathbf{x}}$ operator that we dropped does not.) Moreover, in the space of odd functions of $\mathbf{v}, \delta C$ as given by (2.2) reduces to simply $\delta C=\gamma_{\mathrm{g}}$. So, since $\delta C$ is a symmetric operator, and since it acts to the left on the odd function $\mathbf{v}$ in (2.7), we can replace $(\delta C)^{-1}$ by $\gamma_{\mathrm{g}}^{-1}$ in that equation to obtain

$$
\mathbf{J}=\frac{m_{\mathrm{D}}^{2}}{\gamma_{\mathrm{g}}}\langle\mathbf{v}(\mathbf{E} \cdot \mathbf{v}+\xi)\rangle_{\mathbf{v}}=\sigma \mathbf{E}+\boldsymbol{\zeta}
$$

where 


$$
\boldsymbol{\zeta} \equiv 3 \sigma\langle\mathbf{v} \xi\rangle_{\mathbf{v}}
$$

Using the correlation (2.4) for $\xi$, one produces the correlation (1.3) asserted earlier for $\boldsymbol{\zeta}$. Taking the spatial part of the Maxwell equations (2.1b) and dropping the $d \mathbf{E} / d t$ term which, for the scales (1.1) of interest, is smaller (by four powers of coupling) than the $\sigma \mathbf{E}$ term, one obtains Bödeker's final effective theory (1.2).

\section{LONGITUDINAL SUBTLETIES}

In the introduction, we noted that Bödeker's effective theory (1.2) contains a fluctuating longitudinal electric field. This may seem puzzling since longitudinal electric fields are Debye screened. In this section, we will take a closer look at how both Debye screening, and Bödeker's effective Langevin equation, do emerge from the Boltzmann-Vlasov equations (2.1).

\section{A. Zero mode of $\delta C$}

In the last section, the Boltzmann equation for $W$ was simplified, at leading-log order, by arguing that $\delta C$ dominates over the convective derivative $D_{t}+\mathbf{v} \cdot \mathbf{D}_{\mathbf{x}}$ by a power of $\ln \left(g^{-1}\right)$. This is not quite correct, however, because the operator $\delta C$ has an eigenvalue which is not order $\gamma_{\mathrm{g}}$ and which does not dominate over the convective derivative; specifically, $\delta C$ has a zero mode.

The necessity of this zero mode was noted by Bödeker, who observed that the effective Maxwell equation (2.1D) for the soft fields requires conservation $D_{\mu} J^{\mu}=0$ of the current $J^{\mu}=m_{\mathrm{D}}^{2}\left\langle v^{\mu} W(\mathbf{v})\right\rangle_{\mathbf{v}}$ generated by the hard particles. From (2.1a), this conservation requires $\langle\delta C[W]\rangle_{\mathrm{v}}=0$, which is indeed satisfied by (2.2).

The fact $\langle\delta C[W]\rangle_{\mathbf{v}}=0$ can be rephrased to say that the symmetric operator $\delta C$ has null states: it annihilates anything that is independent of $\mathbf{v}$. (This can be written in braket notation in $\mathbf{v}$-space as $\langle$ constant $|\delta C| W\rangle=\langle W|\delta C|$ constant $\rangle=0$ for any $W$.) This point will be important later on, so let us give an alternative way of understanding it. The collision term $\delta C$ does not care, at leading-log order, about the dynamics of the soft fields. In particular, it does not care that the soft effective theory is a gauge theory, with a local color symmetry, instead of a non-gauge theory, with merely a global color symmetry. So, from the point of view of the calculation of $\delta C$ at leading-log order, the theory could have been one where it was meaningful to talk about the total color charge of the system. If one then imagined adding an infinitesimal chemical potential $\mu$ for this total color charge, the resulting equilibrium density would be

$$
n=\left[e^{\beta\left(\epsilon_{\mathbf{p}}-g \mu_{a} T^{a}\right)} \mp 1\right]^{-1}=n_{0}+n_{0}\left(1 \pm n_{0}\right) \beta g \mu_{a} T^{a}+O\left(\mu^{2}\right)
$$

for each particle type, where $n_{0}$ is the $\mu=0$ equilibrium distribution. In equilibrium, the collision term in a Boltzmann equation always vanishes by detailed balance. Different values of $\mu$ correspond to different equilibrium states, and the collision term must therefore vanish for all $\mu$. That means that the linearized deviation 


$$
\delta n=n_{0}\left(1 \pm n_{0}\right) \beta g \mu_{a} T^{a}
$$

of the equilibrium distribution (3.1) away from $n_{0}$ must correspond to a null state of the linearized collision operator $\delta C$. The deviation (3.2) is isotropic and homogeneous - it is independent of both $\mathbf{v}$ and $\mathbf{x}$. As a result, when re-expressed in terms of the function $W(\mathbf{x}, \mathbf{v})$ used to parametrize color distributions of the hard particles in the linearized Boltzmann equation (2.1a), the deviation (3.2) corresponds to $W(\mathbf{x}, \mathbf{v})=$ constant. That means that a constant $W$ is a null vector of $\delta C$. But since collisions are local in $\mathbf{x}$ (in the effective theory), the $\mathbf{x}$ dependence of $W$ is irrelevant, and so any $W$ which does not depend on $\mathbf{v}$ is a null vector of the linearized collision operator $\delta C$.

\section{B. Longitudinal and transverse projections}

Before continuing, it is worthwhile to introduce longitudinal and transverse projection operators. Perturbatively, the longitudinal projection operator for the electric field is

$$
\left(P_{\mathrm{L}}^{\mathrm{pert}}\right)^{i j}=\hat{k}^{i} \hat{k}^{j}=\nabla^{i} \frac{1}{\nabla^{2}} \nabla^{j}
$$

The gauge-covariant non-perturbative generalization is

$$
P_{\mathrm{L}}^{i j} \equiv D^{i} \frac{1}{D^{2}} D^{j}
$$

where $D^{2}$ means $\mathbf{D} \cdot \mathbf{D}$. The transverse projection operator is of course

$$
P_{\mathrm{T}}^{i j}=\delta^{i j}-P_{\mathrm{L}}^{i j}
$$

It is the longitudinal electric field which couples to external charges, since Gauss' Law reads $\mathbf{D} \cdot \mathbf{E}=\rho$ and since $\mathbf{D} \cdot\left(P_{\mathrm{T}} \mathbf{E}\right)=0$. And it's the transverse electric field that is produced by $\mathbf{D} \times \mathbf{B}$ in the effective theory (1.2) since $P_{\mathrm{L}}(\mathbf{D} \times \mathbf{B})=0$. As mentioned in the introduction, the precise separation between longitudinal and transverse dynamics is not transparent from

this simple discussion because of the non-linear dependence of $\mathbf{D} \times \mathbf{B}$ on the underlying vector potential A.

\section{Solving for $W$ (again)}

To examine the difficulties caused by the presence of a zero mode of $\delta C$, we now return to the effective Boltzmann-Vlasov equation (2.1) and will repeat the analysis of section 【IB, this time being more careful about how we treat the convective derivative compared to $\delta C$. Formally, the solution for $W$ is

$$
W=\frac{1}{D_{t}+\mathbf{v} \cdot \mathbf{D}_{\mathbf{x}}+\delta C}(\mathbf{v} \cdot \mathbf{E}+\xi),
$$

or $W=G(\mathbf{v} \cdot \mathbf{E}+\xi)$, where $G$ denotes the inverse of the linearized kinetic operator,

$$
G \equiv\left[D_{t}+\mathbf{v} \cdot \mathbf{D}_{\mathbf{x}}+\delta C\right]^{-1} .
$$


This fluctuation in the distribution of hard excitations produces a current response [from Eq. (2.1b)] of

$$
\mathbf{J}=m_{\mathrm{D}}^{2}\langle\mathbf{v} G(\mathbf{v} \cdot \mathbf{E}+\xi)\rangle_{\mathbf{v}},
$$

and the (color) charge density

$$
\mathbf{D} \cdot \mathbf{E}=J^{0}=m_{\mathrm{D}}^{2}\langle G(\mathbf{v} \cdot \mathbf{E}+\xi)\rangle_{\mathbf{v}} .
$$

One may easily check that the current is conserved (as it must be), since

$$
\begin{aligned}
D_{0} J^{0}+\mathbf{D} \cdot \mathbf{J} & =m_{\mathrm{D}}^{2}\left\langle\left(D_{t}+\mathbf{v} \cdot \mathbf{D}\right) G(\mathbf{v} \cdot \mathbf{E}+\xi)\right\rangle \\
& =m_{\mathrm{D}}^{2}\langle[1-\delta C G](\mathbf{v} \cdot \mathbf{E}+\xi)\rangle \\
& =0 .
\end{aligned}
$$

The $\langle\mathbf{v} \cdot \mathbf{E}+\xi\rangle_{\mathbf{v}}$ term vanishes due to isotropy, $\langle\mathbf{v}\rangle_{\mathbf{v}}=0$, and the lack of bias in the noise, $\langle\xi\rangle_{\mathbf{v}}=0$. And $\langle\delta C G(\mathbf{v} \cdot \mathbf{E}+\xi)\rangle_{\mathbf{v}}$ vanishes because $\delta C$ is acting (to the left) on its $\mathbf{v}$-independent zero-mode. Đ

\section{The problem with the naive derivation}

In following subsections, we will discuss how to evaluate the operator inverse that defines $G$. To begin, however, it is useful to see how the zero-mode problem manifests itself in a simple example of matrix inversion. To this end, let us for the moment replace the Green function $G$ of (3.7) by that of a simplified finite dimensional example. First, imagine that the gauge interactions are Abelian, so that $D_{t}$ and $\mathbf{D}_{\mathbf{x}}$ can be replaced by simply $-i \omega$ and $i \mathbf{k}$, respectively. Next, imagine that the infinite-dimensional space of possible functions of $\mathbf{v}$, on which $\delta C$ acts, is truncated to the four-dimensional space of functions that are either independent of $\mathbf{v}$ or linear in $\mathbf{v}$. We wish to examine the matrix representing the action of $-i \omega+\mathbf{v} \cdot \mathbf{D}_{\mathbf{x}}+\delta C$ within this truncated space. In order to distinguish clearly between longitudinal and transverse physics, it is convenient to choose a basis $\left\{f_{\alpha}(\mathbf{v})\right\}, \alpha=0, \ldots, 3$, where

$$
f_{0}(\mathbf{v})=1, \quad f_{i}(\mathbf{v})=\sqrt{3} \hat{e}_{i} \cdot \mathbf{v},
$$

and $\hat{e}_{i}$ are three mutually orthonormal unit vectors with $\hat{e}_{1} \equiv \hat{k}$ pointing in the direction of k. The overall normalization has been chosen so that $\left\langle f_{i} f_{j}\right\rangle_{\mathbf{v}}=\delta_{i j}$. In this basis, the matrix elements of $\left\langle f_{i}\left|-i \omega+\mathbf{v} \cdot \mathbf{D}_{\mathbf{x}}+\delta C\right| f_{j}\right\rangle_{\mathbf{v}}$ are

$$
\left(\begin{array}{cccc}
-i \omega & \frac{i}{\sqrt{3}} k & & \\
\frac{i}{\sqrt{3}} k & \gamma_{\mathrm{g}} & & \\
& & -i \omega+\gamma_{\mathrm{g}} & \\
& & & -i \omega+\gamma_{\mathrm{g}}
\end{array}\right)
$$

\footnotetext{
${ }^{6}$ In slightly more explicit notation, this term is $\gamma_{\mathbf{g}}\left\langle\mathcal{I}\left(\mathbf{v}^{\prime \prime}, \mathbf{v}^{\prime}\right) G\left(\mathbf{v}^{\prime}, \mathbf{v}\right)[\mathbf{v} \cdot \mathbf{E}+\xi(\mathbf{x}, \mathbf{v})]\right\rangle_{\mathbf{v}, \mathbf{v}^{\prime}, \mathbf{v}^{\prime \prime}}$. It vanishes because $\left\langle\mathcal{I}\left(\mathbf{v}^{\prime \prime}, \mathbf{v}^{\prime}\right)\right\rangle_{\mathbf{v}^{\prime \prime}}=\left\langle\mathcal{I}\left(\mathbf{v}^{\prime \prime}, \mathbf{v}^{\prime}\right)\right\rangle_{\mathbf{v}^{\prime}}=0$.
} 
The inverse operator, corresponding to $G$ in our truncated space, is

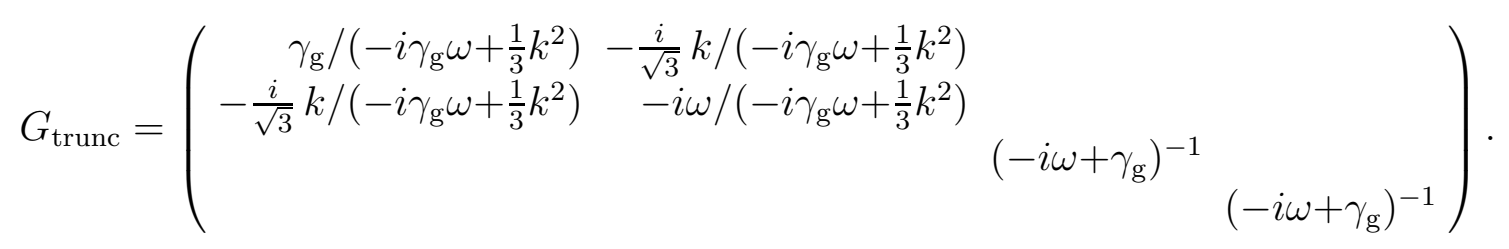

The $\omega \rightarrow 0$ limit is particularly simple:

$$
G_{\text {trunc }} \rightarrow\left(\begin{array}{cccc}
\frac{3 \gamma_{\mathrm{g}}}{k^{2}} & -\frac{i \sqrt{3}}{k} & & \\
-\frac{i \sqrt{3}}{k} & 0 & & \\
& & \gamma_{\mathrm{g}}^{-1} & \\
& & & \gamma_{\mathrm{g}}^{-1}
\end{array}\right)
$$

In contrast, the naive derivation of Bödeker's theory corresponds to replacing $-i \omega+i \mathbf{v} \cdot \mathbf{k}+\delta C$ by $\delta C$, and the corresponding "inverse" would then be

$$
\left(\begin{array}{llll}
\infty & & & \\
& \gamma_{\mathrm{g}}^{-1} & & \\
& & \gamma_{\mathrm{g}}^{-1} & \\
& & & \gamma_{\mathrm{g}}^{-1}
\end{array}\right)
$$

As one can see, there is no difference in the transverse sector (spanned by $f_{2,3}$ ), but there is a huge difference in the longitudinal sector. As a particular example, consider the noiseless part of the current $\mathbf{J}$, given in Eq. 3.8; namely $m_{\mathrm{D}}^{2}\langle\mathbf{v} G \mathbf{v} \cdot \mathbf{E}\rangle_{\mathbf{v}}$. In the naive derivation, as represented by (3.15), this contribution gives

$$
m_{\mathrm{D}}^{2}\left\langle\mathbf{v} \gamma_{\mathrm{g}}^{-1} \mathbf{v} \cdot \mathbf{E}\right\rangle_{\mathbf{v}}=\frac{m_{\mathrm{D}}^{2}}{3 \gamma_{\mathrm{g}}} \mathbf{E}
$$

In our Abelian truncated-space calculation of $G$, however, it is instead given by

$$
\frac{m_{\mathrm{D}}^{2}}{3}\left(G_{11} P_{\mathrm{L}}+G_{22} P_{\mathrm{T}}\right) \mathbf{E}=\frac{m_{\mathrm{D}}^{2}}{3 \gamma_{\mathrm{g}}} P_{\mathrm{T}} \mathbf{E} .
$$

in the $\omega \rightarrow 0$ limit. The longitudinal part of $\mathbf{E}$ is projected out! This is quite different from the result of the naive derivation.

\section{E. Low-frequency, long-wavelength dynamics}

We will now show that, despite the major difference in $G^{-1}$, one nonetheless does recover Bödeker's effective theory even for longitudinal physics. To do so, we will also return to the full, original non-Abelian problem and dispense with the truncated Abelian model of the previous section.

If we restrict our attention to frequencies and wavenumbers which are small compared to the damping rate, $\omega, k \ll \gamma_{\mathrm{g}}$, then in the Greens' function $G$ we could drop the convective derivative compared to $\delta C$ were it not for the fact that $\delta C$ has a zero-mode. To deal with this, let $P_{0}$ denote the projection operator onto the zero-mode of $\delta C$, so that 


$$
P_{0}(f(\mathbf{v})) \equiv\langle f(\mathbf{v})\rangle_{\mathbf{v}},
$$

and separate the convective derivative into zero-mode and non-zero-mode pieces,

$$
D_{t}+\mathbf{v} \cdot \mathbf{D}=\left(D_{t}+\mathbf{v} \cdot \mathbf{D}\right) P_{0}+P_{0} \mathbf{v} \cdot \mathbf{D}+\left(1-P_{0}\right)\left(D_{t}+\mathbf{v} \cdot \mathbf{D}\right)\left(1-P_{0}\right) .
$$

To see this, note that $D_{t}$ commutes with $P_{0}$, and that $P_{0} \mathbf{v} \cdot \mathbf{D} P_{0}=0$. The last term of (3.19) only perturbs the non-zero eigenvalues of $\delta C$, and may be neglected provided $\omega$ and $k$ are small compared to $\gamma_{\mathrm{g}}$. [For $k=O\left(g^{2} T\right)$ this is a leading-log approximation.] The first two terms of (3.19) are rank one perturbations which will lift the zero-mode of the linearized kinetic operator. And one may evaluate explicitly the change in the inverse of an operator produced by adding a finite rank perturbation. In this case, a short exercise shows that

$$
\begin{aligned}
G & \simeq\left[\left(D_{t}+\mathbf{v} \cdot \mathbf{D}\right) P_{0}+P_{0} \mathbf{v} \cdot \mathbf{D}+\delta C\right]^{-1} \\
& =\left(1-P_{0}\right) \delta C^{-1}\left(1-P_{0}\right)+\gamma_{\mathrm{g}}^{-1}\left(\gamma_{\mathrm{g}}-\mathbf{v} \cdot \mathbf{D}\right) \frac{P_{0}}{\gamma_{\mathrm{g}} D_{t}-\frac{1}{3} \mathbf{D}^{2}}\left(\gamma_{\mathrm{g}}-\mathbf{v} \cdot \mathbf{D}\right)
\end{aligned}
$$

(To verify the last equality, recall that $\delta C$ is nothing but multiplication by $\gamma_{\mathrm{g}}$ when acting on odd functions of $\mathbf{v}$. Hence, $\delta C \mathbf{v} \cdot \mathbf{D} P_{0}=\gamma_{\mathrm{g}} \mathbf{v} \cdot \mathbf{D} P_{0}$.)

Now pause to note the correspondences of this result with the truncated Abelian results of the previous section. The full inverse $(3.20)$ gives

$$
\begin{aligned}
\langle G\rangle_{\mathbf{v}} & \simeq \gamma_{\mathrm{g}}\left[\gamma_{\mathrm{g}} D_{t}-\frac{1}{3} \mathbf{D}^{2}\right]^{-1} \\
\langle\mathbf{v} G\rangle_{\mathbf{v}} & \simeq \frac{1}{3} \mathbf{D}\left[\gamma_{\mathrm{g}} D_{t}-\frac{1}{3} \mathbf{D}^{2}\right]^{-1} \\
\langle G \mathbf{v}\rangle_{\mathbf{v}} & \simeq\left[\gamma_{\mathrm{g}} D_{t}-\frac{1}{3} \mathbf{D}^{2}\right]^{-1} \frac{1}{3} \mathbf{D} \\
\langle\mathbf{v} G \mathbf{v}\rangle_{\mathbf{v}} & \simeq \gamma_{\mathrm{g}}\left\{1-\frac{1}{3} \mathbf{D}\left[\gamma_{\mathrm{g}} D_{t}-\frac{1}{3} \mathbf{D}^{2}\right]^{-1} \frac{1}{3} \mathbf{D}\right\} \underset{D_{t} \rightarrow 0}{\longrightarrow} \frac{1}{3 \gamma_{\mathrm{g}}} P_{\mathrm{T}}
\end{aligned}
$$

for $\omega, k \ll \gamma_{\mathrm{g}}$. These are simple non-Abelian generalizations of (3.13). (The factor of 3 differences just reflect the normalizations of our chosen basis functions in the last section.)

The form (3.20) for $G$ may now be inserted into Eqs. (3.8) and (3.9). For the charge density, one finds

$$
\mathbf{D} \cdot \mathbf{E}=J^{0}=-\gamma_{\mathrm{g}}\left[\gamma_{\mathrm{g}} D_{t}-\frac{1}{3} \mathbf{D}^{2}\right]^{-1}(\sigma \mathbf{D} \cdot \mathbf{E}+\mathbf{D} \cdot \boldsymbol{\zeta})
$$

or

$$
\left[D_{t}+\frac{\sigma}{m_{\mathrm{D}}^{2}}\left(-\mathbf{D}^{2}+m_{\mathrm{D}}^{2}\right)\right] \mathbf{D} \cdot \mathbf{E}=-\mathbf{D} \cdot \boldsymbol{\zeta},
$$

where $\sigma \equiv \frac{1}{3} m_{\mathrm{D}}^{2} / \gamma_{\mathrm{g}}$, and $\boldsymbol{\zeta} \equiv 3 \sigma\langle\mathbf{v} \xi\rangle_{\mathbf{v}}$. And for the current, from (3.8) and (3.22),

$$
\mathbf{J}=\sigma \mathbf{E}+\boldsymbol{\zeta}-\frac{\sigma}{m_{\mathrm{D}}^{2}} \mathbf{D}(\mathbf{D} \cdot \mathbf{E})
$$

Inserting this into the Maxwell equation $-D_{t} \mathbf{E}+\mathbf{D} \times \mathbf{B}=\mathbf{J}$ gives 


$$
D_{t} \mathbf{E}+\sigma \mathbf{E}-\frac{\sigma}{m_{\mathrm{D}}^{2}} \mathbf{D}(\mathbf{D} \cdot \mathbf{E})=\mathbf{D} \times \mathbf{B}-\boldsymbol{\zeta}
$$

This local equation of motion is the exact result which follows from approximating $G$ as shown in (3.20). However, that form for $G$ was based on the assumption that the frequencies and wavevectors of interest are small compared to the damping rate $\gamma_{\mathrm{g}}$. Since $\gamma_{\mathrm{g}}$ is $O\left(g^{2} T \ln g^{-1}\right)$, this means that $\omega$ is tiny compared to the $O\left(T / \ln g^{-1}\right)$ conductivity, and that $k$ is much smaller than the $O(g T)$ Debye mass. Hence, there is no point in retaining the $D_{t} \mathbf{E}$ or $\mathbf{D}(\mathbf{D} \cdot \mathbf{E})$ terms in the effective equation (3.25). Dropping these terms immediately yields Bödeker's equation (1.2). In other words, a more careful treatment of the effect of the zero mode in $\delta C$ does not produce any difference (in leading-logarithmic approximation) to the resulting effective theory.

\section{F. Recovering Debye screening}

To see how Debye screening emerges from the kinetic theory (2.1), return to Eq. (3.6) for $W$ and now assume that the scales of interest are in the perturbative regime where $k \gg g^{2} T$ and/or $\omega \gg g^{4} T \ln g^{-1}$. [In other words, the necessary conditions (1.1b) for non-perturbative fluctuations are not both satisfied.] In this regime, the gauge fields in the covariant derivatives appearing in the Greens' function $G$ may be treated as small.ป Expanding $G$ in powers of the gauge field, the leading term,

$$
G \simeq\left[\partial_{t}+\mathbf{v} \cdot \nabla_{\mathbf{x}}+\delta C\right]^{-1}
$$

is diagonal in momentum space. Fourier transforming Eq. (3.9) for the charge density then yields (to leading order in the gauge field)

$$
\begin{aligned}
i \mathbf{k} \cdot \mathbf{E} & =m_{\mathrm{D}}^{2}\langle\tilde{G} \mathbf{v}\rangle_{\mathbf{v}} \cdot \mathbf{E}+m_{\mathrm{D}}^{2}\langle\tilde{G} \xi\rangle_{\mathbf{v}} \\
& =m_{\mathrm{D}}^{2}\langle\tilde{G} \mathbf{v} \cdot \mathbf{k}\rangle_{\mathbf{v}} \frac{\mathbf{k} \cdot \mathbf{E}}{\mathbf{k}^{2}}+m_{\mathrm{D}}^{2}\langle\tilde{G} \xi\rangle_{\mathbf{v}} \\
& =-i m_{\mathrm{D}}^{2}\left(1+i \omega\langle\tilde{G}\rangle_{\mathbf{v}}\right) \frac{\mathbf{k} \cdot \mathbf{E}}{\mathbf{k}^{2}}+m_{\mathrm{D}}^{2}\langle\tilde{G} \xi\rangle_{\mathbf{v}}
\end{aligned}
$$

or

$$
\left[\mathbf{k}^{2}+m_{\mathrm{D}}^{2}\left(1+i \omega\langle\tilde{G}\rangle_{\mathbf{v}}\right)\right] i \mathbf{k} \cdot \mathbf{E}=m_{\mathrm{D}}^{2} \mathbf{k}^{2}\langle\tilde{G} \xi\rangle_{\mathbf{v}}
$$

with

$$
\tilde{G}(\omega, \mathbf{k})=[-i \omega+i \mathbf{v} \cdot \mathbf{k}+\delta C]^{-1}
$$

and $\mathbf{E}$ and $\xi$ now denoting the $(\omega, \mathbf{k})$ Fourier components of these fields. In the first step of (3.27), we used the fact that, with gauge fields neglected in $\tilde{G}$, the only vector which $\langle G \mathbf{v}\rangle_{\mathbf{v}}$

\footnotetext{
${ }^{7}$ For a more detailed justification, based on a computation of the power spectrum of gauge field fluctuations, see Ref. [1].
} 
can depend upon is $\mathbf{k}$, and therefore only the longitudinal component of $\mathbf{E}$ can contribute to the result. The following step used $\langle\tilde{G}(i \mathbf{v} \cdot \mathbf{k}-i \omega)\rangle=1-\langle\tilde{G} \delta C\rangle=1$ which is another consequence of the zero mode in $\delta C$. The result (3.28) shows that $\mathbf{k} \cdot \mathbf{E}$ satisfies a diffusive Langevin equation in which the noise and damping depend on $\langle\tilde{G} \xi\rangle_{\mathbf{v}}$ and $\langle\tilde{G}\rangle_{\mathbf{v}}$, respectively.

The power spectrum of charge density (or $\mathbf{D} \cdot \mathbf{E}$ ) fluctuations is defined as

$$
\rho_{\mathrm{L}}(\omega, \mathbf{k}) \equiv \int d t d^{3} \mathbf{x} e^{i \omega t-i \mathbf{k} \cdot \mathbf{x}}\left\langle J^{0}(t, \mathbf{x}) J^{0}(0,0)\right\rangle_{\xi}
$$

Using (3.28) to express $\mathbf{D} \cdot \mathbf{E}$ in terms of the noise $\xi$, and then recalling that the covariance of the noise $\xi$, as given by (2.4) and (2.2), is proportional to $\delta C$, allows one to write the power spectrum as

$$
\begin{aligned}
\rho_{\mathrm{L}}(\omega, \mathbf{k}) & =m_{\mathrm{D}}^{4} \mathbf{k}^{4}\left\langle\langle\tilde{G} \xi\rangle_{\mathbf{v}}\left\langle\xi \tilde{G}^{\dagger}\right\rangle_{\mathbf{v}^{\prime}}\right\rangle_{\xi} /\left|\mathbf{k}^{2}+m_{\mathrm{D}}^{2}\left(1+i \omega\langle\tilde{G}\rangle_{\mathbf{v}}\right)\right|^{2} \\
& =2 g^{2} T m_{\mathrm{D}}^{2} \mathbf{k}^{4} \operatorname{Re}\langle\tilde{G}\rangle_{\mathbf{v}} /\left|\mathbf{k}^{2}+m_{\mathrm{D}}^{2}\left(1+i \omega\langle\tilde{G}\rangle_{\mathbf{v}}\right)\right|^{2} \\
& =-\frac{2 T}{\omega} \operatorname{Im} \frac{g^{2} \mathbf{k}^{4}}{\mathbf{k}^{2}+m_{\mathrm{D}}^{2}\left(1+i \omega\langle\tilde{G}\rangle_{\mathbf{v}}\right)}
\end{aligned}
$$

Once again, this answer is valid provided $k \gg g^{2} T$ and/or $\omega \gg g^{4} T \ln g^{-1}$ since we neglected (for this discussion only) the soft gauge fields in the covariant derivatives. Furthermore, $k$ and $\omega$ must be small compared to $T$, since this is a basic requirement for any kinetic theory description to be valid.

Linear response theory, applied to the underlying quantum field theory, shows that the power spectrum

$$
\rho_{\mathrm{L}}(\omega, \mathbf{k}) \equiv \int d t d^{3} \mathbf{x} e^{i \omega t-i \mathbf{k} \cdot \mathbf{x}}\left\langle\frac{1}{2}\left\{J^{0}(t, \mathbf{x}), J^{0}(0,0)\right\}\right\rangle,
$$

(defined with a symmetrized ordering of quantum operators) is related to the retarded charge-density charge-density correlator

$$
D_{\mathrm{R}}(\omega, \mathbf{k}) \equiv i \int d t d^{3} \mathbf{x} e^{i \omega t-i \mathbf{k} \cdot \mathbf{x}} \theta(t)\left\langle\left[J^{0}(t, \mathbf{x}), J^{0}(0,0)\right]\right\rangle,
$$

by the fluctuation-dissipation relation

$$
\rho_{\mathrm{L}}(\omega, \mathbf{k})=[2 n(\omega)+1] \operatorname{Im} D_{\mathrm{R}}(\omega, \mathbf{k}),
$$

where $n(\omega)=\left[e^{\beta \omega}-1\right]^{-1}$ is the equilibrium Bose distribution function. And the retarded correlator $D_{\mathrm{R}}(\omega, \mathbf{k})$ is the analytic continuation of the Euclidean space (time-ordered imaginarytime) correlator $D_{\mathrm{E}}\left(i \omega_{n}, \mathbf{k}\right)$ from the imaginary Matsubara frequencies to (just above) the real frequency axis.

By comparing (3.31) to the form (3.33), the kinetic theory result for the power spectrum may be converted to an equivalent result for the retarded correlator. The leading factor of $T / \omega$ is just the low-frequency (classical) limit of the Bose distribution function, and hence the retarded charge density correlator is

$$
D_{\mathrm{R}}(\omega, \mathbf{k})=g^{2} \mathbf{k}^{2}-\frac{g^{2} \mathbf{k}^{4}}{\mathbf{k}^{2}+m_{\mathrm{D}}^{2}\left(1+i \omega\langle\tilde{G}\rangle_{\mathbf{v}}\right)} .
$$


The local (and temperature-independent) $g^{2} \mathbf{k}^{2}$ term, which does not contribute to the imaginary part, is determined by the current-current Ward identities, or equivalently by the requirement that $D_{\mathrm{R}}$ remain bounded as $\mathbf{k} \rightarrow \infty$. The low frequency limit,

$$
D_{\mathrm{R}}(0, \mathbf{k})=\frac{g^{2} \mathbf{k}^{2} m_{\mathrm{D}}^{2}}{\mathbf{k}^{2}+m_{\mathrm{D}}^{2}}=g^{2}\left[m_{\mathrm{D}}^{2}-\frac{m_{\mathrm{D}}^{4}}{\mathbf{k}^{2}+m_{\mathrm{D}}^{2}}\right]
$$

reproduces the correct static equilibrium Debye-screened charge density correlations. P More generally, the kinetic theory answer (3.35) reduces to the known hard-thermal-loop result whenever the frequency or momentum is large compared to the damping rate $\gamma_{\mathrm{g}}$. In this domain, the details of the scattering operator $\delta C$ are irrelevant, and $\tilde{G}(\omega, \mathbf{k})$ may be approximated by $i[\omega-\mathbf{v} \cdot \mathbf{k}+i \epsilon]^{-1}$. The resulting average over $\mathbf{v}$ may then be performed analytically, and yields the standard HTL result for the self-energy.

If neither $k$ nor $\omega$ are large compared to the damping rate $\gamma_{\mathrm{g}}$, then the detailed form of $\delta C$ is significant. Evaluating $\tilde{G}(\omega, k)$ is non-trivial if $k$ is comparable to the damping rate. However, if $k$ and $\omega$ are both small compared to $\gamma_{\mathrm{g}}$, then the previous representation (3.21a) may be used. Perturbatively, it gives

$$
\langle\tilde{G}(\omega, \mathbf{k})\rangle_{\mathbf{v}}=\frac{\gamma_{\mathrm{g}}}{\frac{1}{3} \mathbf{k}^{2}-i \omega \gamma_{\mathrm{g}}},
$$

which gives

$$
\left.\rho_{\mathrm{L}}(\omega, \mathbf{k})\right|_{\text {Bödeker }}=\frac{2 g^{2} T}{\sigma} \mathbf{k}^{2} .
$$

This is the same result which emerges directly from the relation $\sigma \mathbf{D} \cdot \mathbf{E}=-\mathbf{D} \cdot \boldsymbol{\zeta}$ in Bödeker's effective theory, combined with (1.3) for the noise variance. This result is valid in the overlap of the perturbative and damping-dominated domains, that is when $\left|k^{2}-i \omega \sigma\right| \gg g^{4} T^{2}$ and $\left|\frac{1}{3} k^{2}-i \omega \gamma_{\mathrm{g}}\right| \ll \gamma_{\mathrm{g}}^{2}$.

\section{IRRELEVANCY OF LONGITUDINAL DYNAMICS}

We have seen that Bödeker's effective theory

$$
\sigma \mathbf{E}=\mathbf{D} \times \mathbf{B}-\boldsymbol{\zeta},
$$

within its domain of validity, does correctly describe both longitudinal and transverse fluctuations. However, it is the transverse part of the gauge fields which are responsible for interesting non-perturbative phenomena such as topological transitions and associated baryon non-conservation. One may wonder if it is possible to formulate an equally valid effective

\footnotetext{
${ }^{8}$ The factor of $g^{2}$ is present merely because we have chosen to scale all our gauge fields by $g$ relative to the usual perturbative conventions. In, for example, Coulomb gauge, $D_{\mathrm{R}}$ equals the one-loop gauge field self-energy $\Pi^{00}$ (which is just $m_{\mathrm{D}}^{2}$ in the static limit), plus the one-particle reducible contributions which sum to $\Pi^{00} D^{00} \Pi^{00}$, where $D^{00}=\left\langle A^{0} A^{0}\right\rangle=-1 /\left(\mathbf{k}^{2}+\Pi^{00}\right)$ is the Debye-screened $A^{0}$ propagator.
} 
theory which describes only transverse physics. On the face of things, this should be easy; just insert a transverse projection operator to eliminate the longitudinal part of the noise,

$$
\sigma \mathbf{E}=\mathbf{D} \times \mathbf{B}-P_{\mathrm{T}} \boldsymbol{\zeta}
$$

This produces an effective theory with no longitudinal dynamics whatsoever, $P_{\mathrm{L}} \mathbf{E}=0$. In the case of an Abelian theory, the trivial decoupling of transverse and longitudinal parts of the gauge field would make it obvious that Bödeker's theory (4.1) and the transverseprojected theory (4.2) describe exactly the same transverse dynamics. But for our nonAbelian theory, the dependence of covariant derivatives and projection operators on the gauge field makes this decoupling far less obvious. The remainder of this paper is devoted to showing that it is almost true that equations (4.1) and (4.2) do in fact generate identical transverse dynamics. The "almost" caveat reflects subtleties associated with the fact that white noise cannot be considered smooth even on infinitesimal time scales. Investigating these subtleties will reveal that the naive transverse equation (4.2) must be corrected but can then be made exactly equivalent to Bödeker's equation (4.1) with regard to the transverse dynamics. It should be emphasized that this investigation was motivated by theoretical curiosity, not practical convenience. Pl Bödeker's equation (4.1) is local, whereas the transverse-projected equation (4.2) is not. Consequently, numerical simulations are far more easily performed in the original theory than in any transverse-projected variant.

To be precise, by "transverse dynamics" we refer to all physical observables that do not depend on $A_{0}$ when expressed as gauge-invariant functions of the fields $A_{\mu}$. The magnetic field $\mathbf{B}=\mathbf{D} \times \mathbf{A}$ does not, of course, depend on $A_{0}$. Writing $\mathbf{E}=\mathbf{D} A_{0}-(d \mathbf{A} / d t)$, it is easy to see that the transverse electric field $P_{\mathrm{T}} \mathbf{E}=-P_{\mathrm{T}}(d \mathbf{A} / d t)$ does not either. Consequently, an example of a physical quantity which depends only on the transverse dynamics is the topological charge (or change in Chern-Simons number) of the gauge field, which is proportional $\square$ to $\int \operatorname{tr}[\mathbf{E} \cdot \mathbf{B}]=\int \operatorname{tr}\left[\left(P_{\mathrm{T}} \mathbf{E}\right) \cdot \mathbf{B}\right] \cdot \mathbf{\square}$

\section{A. Naive equivalence}

We first wish to paint with a broad brush. We will for the moment ignore all subtleties and discuss how, if one implicitly and incorrectly (and only when advantageous) treats the noise $\boldsymbol{\zeta}(\mathbf{x}, t)$ as a smooth function of $t$, one may show that the two theories (4.1) and (4.2) should generate the same transverse dynamics. We will wait until section $\mathbb{D B}$ and its sequel

\footnotetext{
9 In fact, historically, it took us some time to recognize that longitudinal physics for $\omega \ll k \ll \gamma_{\mathrm{g}}$ is correctly reproduced by Bödeker's effective theory, and the discussion in this section was motivated by the desire to show that it simply doesn't matter.

10 The precise formula is $\Delta N(t)=\left(1 / 8 \pi^{2}\right) \int_{0}^{t} d t d^{3} x E_{i}^{a} B_{i}^{a}$.

${ }^{11}$ The topological transition rate (or Chern-Simons number diffusion constant), is an important ingredient in scenarios of electroweak baryogenesis. Understanding the applicability of numerical simulations using Bödeker's effective theory for extracting the topological transition rate motivated this investigation. See [9] for such recent numerical work and related discussion.
} 
to correct this discussion by taking into account the non-smooth nature of Gaussian white noise.

It is simplest to initially consider both theories (4.2) and (4.1) in $A_{0}=0$ gauge:

$$
\sigma \frac{d}{d t} \mathbf{A}=-\mathbf{D} \times \mathbf{B}+\boldsymbol{\zeta}
$$

and

$$
\sigma \frac{d}{d t} \mathbf{A}=-\mathbf{D} \times \mathbf{B}+P_{\mathrm{T}} \boldsymbol{\zeta}
$$

For the moment, imagine a particular instantiation of the white noise $\boldsymbol{\zeta}(\mathbf{x}, t)$ - that is, consider a particular member of the Gaussian ensemble of noise functions. Suppose that Bödeker's equation (4.3) is satisfied by a gauge field $\mathbf{A}(\mathbf{x}, t)$. Now rewrite Bödeker's equation in the form

$$
\sigma\left(\frac{d}{d t} \mathbf{A}-\sigma^{-1} P_{\mathrm{L}} \boldsymbol{\zeta}\right)=-\mathbf{D} \times \mathbf{B}+P_{\mathrm{T}} \boldsymbol{\zeta}
$$

Using the explicit form (3.4) of the longitudinal projection operator, this can be written as

$$
\sigma\left(\frac{d}{d t} \mathbf{A}-\mathbf{D} \tilde{A}_{0}\right)=-\mathbf{D} \times \mathbf{B}+P_{\mathrm{T}} \boldsymbol{\zeta},
$$

where $\tilde{A}_{0}$ is simply a (suggestive) name for

$$
\tilde{A}_{0} \equiv \sigma^{-1} D^{-2} \mathbf{D} \cdot \boldsymbol{\zeta}
$$

For a particular instantiation of the noise (and any initial condition), the solution to (4.6) may be interpreted in two different ways. On the one hand, $\mathbf{A}$ is, by construction, an $A_{0}=0$ gauge solution to Bödeker's equation (4.1). On the other hand, if one says that $\tilde{A}_{0}$ is actually the time component of the gauge field, then the left-hand-side of (4.6) is just $-\sigma \mathbf{E}$. Therefore, $A_{\mu}=\left(\tilde{A}_{0}, \mathbf{A}\right)$ is a solution to the projected equation (4.2) in the particular gauge where $A_{0}=\sigma^{-1} D^{-2} \mathbf{D} \cdot \boldsymbol{\zeta}$.

But, given a solution $\left(\tilde{A}_{0}, \mathbf{A}\right)$ to $(4.2)$ with $A_{0} \neq 0$, one may always gauge transform back to $A_{0}=0$ gauge. The result will be a gauge field $\overline{\mathbf{A}}$ which obeys

$$
\sigma \frac{d}{d t} \overline{\mathbf{A}}=-\overline{\mathbf{D}} \times \overline{\mathbf{B}}+P_{\mathrm{T}} \overline{\boldsymbol{\zeta}}
$$

This is just the $A_{0}=0$ transverse equation (4.4), except that the noise has been gauge transformed by the transformation which takes $\left(A_{0}, \mathbf{A}\right)$ into $A_{0}=0$ gauge:

$$
\bar{\zeta}^{a}=U^{a b} \zeta^{b}
$$

with

$$
U=\mathcal{T} \exp \left[\int_{0}^{t} \tilde{A}_{0} d t\right]=\mathcal{T} \exp \left[\sigma^{-1} \int_{0}^{t} \frac{1}{D^{2}} \mathbf{D} \cdot \boldsymbol{\zeta} d t\right]
$$


where $\mathcal{T}$ signifies time ordering, with the latest times on the right.

The distinction between $\boldsymbol{\zeta}$ and its gauge transform $\bar{\zeta}$ will not matter, and our two theories (4.3) and (4.4) will be equivalent (subject to earlier caveats), provided the distribution $\overline{\boldsymbol{\zeta}}^{a}=U^{a b} \boldsymbol{\zeta}^{b}$ is Gaussian white noise, just like the distribution of the original $\boldsymbol{\zeta}$. If our transformation $U$ was not a function of the noise, this would be trivial because then we would have

$$
\left\langle\overline{\boldsymbol{\zeta}}^{a} \overline{\boldsymbol{\zeta}}^{b}\right\rangle=U^{a c} U^{b d}\left\langle\boldsymbol{\zeta}^{c} \boldsymbol{\zeta}^{d}\right\rangle
$$

Since the $\boldsymbol{\zeta}$ correlator is proportional to $\delta^{c d}$, and since $U^{a c} U^{b d} \delta^{c d}=\delta^{a b}$, it would follow that

$$
\left\langle\overline{\boldsymbol{\zeta}}^{a} \overline{\boldsymbol{\zeta}}^{b}\right\rangle=\left\langle\boldsymbol{\zeta}^{a} \boldsymbol{\zeta}^{b}\right\rangle
$$

Even though our transformation $U$ depends on the Gaussian white noise $\boldsymbol{\zeta}$, this result naively remains true. Consider, for instance, the equal time correlation

$$
\left\langle\overline{\boldsymbol{\zeta}}^{a}(t) \overline{\boldsymbol{\zeta}}^{b}(t)\right\rangle=\left\langle U^{a c}(t) U^{b d}(t) \boldsymbol{\zeta}^{c}(t) \boldsymbol{\zeta}^{d}(t)\right\rangle .
$$

Because the noise correlation is local in time, while $U$ (formally) depends only on the noise prior to $t$, this can be factorized:프

$$
\left\langle\overline{\boldsymbol{\zeta}}^{a}(t) \overline{\boldsymbol{\zeta}}^{b}(t)\right\rangle=\left\langle U^{a c}(t) U^{b d}(t)\right\rangle\left\langle\boldsymbol{\zeta}^{c}(t) \boldsymbol{\zeta}^{d}(t)\right\rangle \text {. }
$$

The $\boldsymbol{\zeta}$ correlation is again proportional to $\delta^{c d}$, which again contracts the $U$ 's and eliminates them, so that we arrive at (4.12) as desired. A similar argument shows that unequal time correlations of $\overline{\boldsymbol{\zeta}}$ vanish, as they should.

\section{B. So what's wrong?}

\section{A toy model}

To see what goes wrong with the previous equivalency argument, and to understand what it has to do with the short-time nature of white noise, it is instructive first to consider a system much simpler than non-Abelian gauge theory. Forget about field theory and instead imagine stochastic dynamics of a classical particle moving in two dimensions in a rotationally-symmetric potential $V(r)$ :

$$
\begin{gathered}
\frac{d}{d t} \mathbf{r}=-\nabla V+\boldsymbol{\zeta}, \\
\left\langle\zeta_{i}(t) \zeta_{j}\left(t^{\prime}\right)\right\rangle=2 T \delta_{i j} \delta\left(t-t^{\prime}\right) .
\end{gathered}
$$

\footnotetext{
${ }^{12}$ In fact, the dependence (or lack thereof) of $U(t)$ on the noise at exactly time $t$ is ill-defined and depends on the details of time discretization, as discussed in the next sub-section.
} 
For convenience, we have normalized the analog of $\sigma$ to 1 . Imagine also we care only about the radial dynamics of this system and not at all about the angular dynamics.

Comparing to the gauge theory problem, $\mathbf{r}$ above is analogous to $\mathbf{A}$ in $A_{0}=0$ gauge, the radial dynamics to the transverse gauge dynamics, and the angular dynamics to the longitudinal dynamics. Circles about the origin are analogous to gauge orbits of 3-dimensional gauge configurations under 3-dimensional gauge transformations (since, in the gauge theory, infinitesimal displacements in the longitudinal direction are of the form $\Delta \mathbf{A}(\mathbf{x})=\mathbf{D} \Lambda(\mathbf{x})$, which is the form of an infinitesimal 3-dimensional gauge transformation). Eq. (4.15) is analogous to Bödeker's effective theory (4.3), and the analog of the transverse-projected theory (4.4) is then

$$
\frac{d}{d t} \mathbf{r}=-\nabla V+P_{\mathrm{r}} \boldsymbol{\zeta}
$$

where the radial projection operator $P_{\mathrm{r}}$ is

$$
P_{\mathrm{r}}^{i j}=\hat{r}^{i} \hat{r}^{j}=\delta^{i j}-\hat{\theta}^{i} \hat{\theta}^{j}
$$

Just as in section $\mathbb{E A}$, we can make a sloppy and not quite correct argument that the unprojected equation (4.15) and the projected equation (4.17) are equivalent. A transformation from a solution $\mathbf{r}$ of the unprojected equation to a solution $\overline{\mathbf{r}}$ of the projected equation appears to be

$$
\overline{\mathbf{r}}=U \mathbf{r}, \quad \bar{\zeta}=U \boldsymbol{\zeta}
$$

or equivalently

$$
\mathbf{r}=U^{-1} \overline{\mathbf{r}}, \quad \boldsymbol{\zeta}=U^{-1} \overline{\boldsymbol{\zeta}},
$$

where, if $\mathbf{r}$ and $\boldsymbol{\zeta}$ are represented by complex numbers $r_{x}+i r_{y}$ and $\zeta_{x}+i \zeta_{y}, U$ can be written in a form quite analogous to (4.10):

$$
U=\exp \left(-i \int_{0}^{t} \hat{\theta} \cdot \zeta d t\right)
$$

$U$ simply rotates away the accumulated motion in the angular direction, so that the projected motion, at every instant in time, becomes purely radial. Naively plugging (4.20) into the unprojected equation (4.15), and implicitly but incorrectly assuming that $\boldsymbol{\zeta}$ is a smooth function of time, yields the naive projected equation (4.17) for $\overline{\mathbf{r}}$.

One can immediately see that the two equations (4.15) and (4.17) cannot, however, actually describe the same radial dynamics. In the unprojected case (4.15), there is zero probability that the system would ever pass exactly through the origin $r=0$. The projected case (4.17), however, just describes one-dimensional motion, parameterized by $r$, along a line of constant $\theta$. That is, we could fix $\theta$ and just replace (4.17) by the one degree of freedom equation

$$
\frac{d}{d t} r=-\frac{d V}{d r}+\zeta
$$


(There does not appear to be an analog of this simplification in the gauge theory; see Appendix B.) As long as there are no infinite potential barriers, this one-dimensional system will eventually fluctuate through any value of $r$, including $r=0$.

To understand the discrepancy, we need to properly understand the small time behavior of white noise Langevin equations such as (4.15) and (4.17). The standard way of defining what such equations actually mean is to discretize time and only at the end of the day take the continuous time limit.

\section{Time discretization ambiguities}

Before proceeding, we have to dispose of an instructive red herring concerning the time discretization of our various stochastic equations. It is not always true that continuum-time stochastic equations like the ones we have been writing down have an unambiguous meaning. To understand the possible ambiguities, imagine that instead of being interested in only the radial dynamics of our toy model, we were instead interested in only the angular dynamics, and so had proposed a projected equation of the form

$$
\begin{gathered}
\frac{d}{d t} \mathbf{r}=P_{\theta} \boldsymbol{\zeta}, \\
P_{\theta}^{i j}=\hat{\theta}^{i} \hat{\theta}^{j}=\delta^{i j}-\hat{r}^{i} \hat{r}^{j} .
\end{gathered}
$$

This continuum equation appears to describe motion for which the radius $r$ remains constant. Now imagine discretizing time with small time steps of size $\epsilon$, so that

$$
\begin{gathered}
\epsilon^{-1} \Delta \mathbf{r}=P_{\theta} \boldsymbol{\zeta}, \\
\left\langle\zeta_{i}(t) \zeta_{j}(t+n \epsilon)\right\rangle=2 T \epsilon^{-1} \delta_{i j} \delta_{n 0} .
\end{gathered}
$$

There is an ambiguity in the schematic way we have written the discretized equation (4.25): we have not made it clear whether the direction $\hat{\theta}$ implicit in $P_{\theta}$ is supposed to be evaluated at the starting point of the tiny time interval, the end point, or somewhere in between. In the first case, the value of $r$ will drift out a little bit, as in fig. 1a. In the second case, it will drift in a little bit, as in fig. [7b. If we pick a symmetric convention, where we evaluate $\hat{\theta}$ at the midpoint, then $r$ will remain constant, as in fig. 1 $\mathrm{c}$.

In non-stochastic equations, such discretization choices become irrelevant in the continuum limit $\epsilon \rightarrow 0$ (though they may have significance for the practicality of numerical calculations). For stochastic equations, however, the $\epsilon \rightarrow 0$ limit is more subtle because, by (4.26), the amplitude of the white noise $\boldsymbol{\zeta}$ is order

$$
\zeta \sim \sqrt{\frac{T}{\epsilon}}
$$

and diverges as $\epsilon \rightarrow 0$. The drift $\Delta r$ in figs. 1 $1 \mathrm{a}$ and $\mathrm{b}$ is therefore of order

$$
\pm \Delta r \sim \sqrt{r^{2}+(\epsilon \zeta)^{2}}-r \sim \frac{(\epsilon \zeta)^{2}}{r} \sim \frac{\epsilon T}{r}
$$




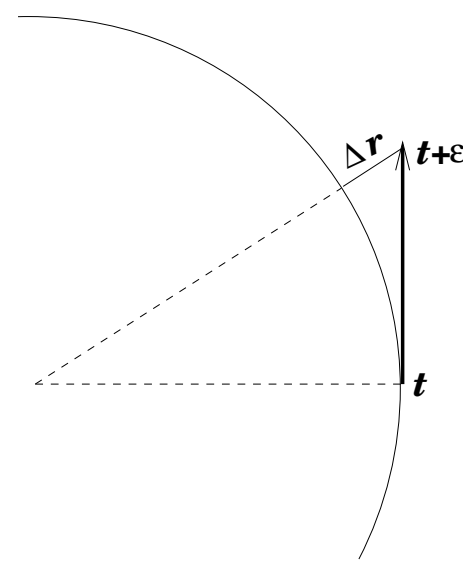

(a)

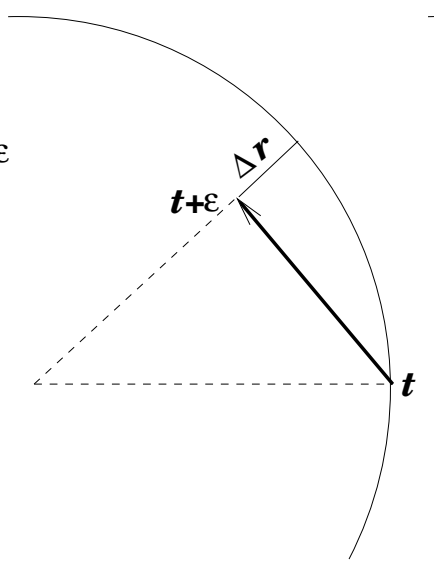

(b)

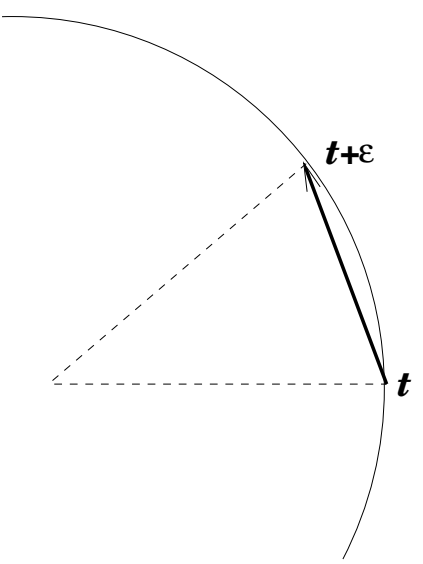

(c)

FIG. 1. Radial motion generated by (4.25) depending on whether $\hat{\theta}$ is defined by the (a) beginning, (b) end, or (c) middle of a discrete time jump $t \rightarrow t+\epsilon$.

for a time interval $\epsilon$. That means that the drift per unit time, $\Delta r / \epsilon$, is finite as $\epsilon \rightarrow 0$, and so the continuum limit really depends on one's discretization conventions.

In the unprojected equation (4.15), there is no such discretization ambiguity. And in our actual toy model equation (4.17) with radial projection, there is no such ambiguity because motion in the $\hat{\mathbf{r}}$ direction, unlike in the $\hat{\theta}$ direction, is straight $-\hat{\mathbf{r}}$ does not change between one end of the interval and the other. The situation is slightly more complicated for the transverse-projected equation (4.4) for gauge theory, however. There, motion of A in the transverse direction does change the transverse projection operator $P_{\mathrm{T}}$. However, we demonstrate in section IVC2 that this change turns out to be high enough order in $\delta \mathbf{A}$ that discretization ambiguities do not arise.

The upshot is that the continuum stochastic equations (4.17) and (4.4) for the radialprojected toy model and the transverse-projected gauge theory are not ambiguous. However, we shall next see that the very same discretization issues affect the transformations we used to argue that they were equivalent with their unprojected counterparts.

\section{Centrifugal drift}

The way we proposed obtaining the projected equation (4.17) from the unprojected one (4.15) was by rotating away the accumulated $\theta$ motion. Imagine a single time step of the discretized unprojected equation. Then [3]

$$
\mathbf{r}(t+\epsilon)=\mathbf{r}(t)-\epsilon \boldsymbol{\nabla} V(r(t))+\epsilon \boldsymbol{\zeta}(t) .
$$

The motion of the radial coordinate $r$ is then

\footnotetext{
13 Whether it's $V(r(t))$ or $V(r(t+\epsilon))$, or the average of the two, does not matter in the continuum time limit $\epsilon \rightarrow 0$.
} 


$$
\begin{aligned}
r(t+\epsilon) & =|\mathbf{r}(t)-\epsilon \boldsymbol{\nabla} V(r(t))+\epsilon \boldsymbol{\zeta}(t)| \\
& =\left.\left[r-\epsilon V^{\prime}(r)+\epsilon \hat{\mathbf{r}} \cdot \boldsymbol{\zeta}+\frac{\epsilon^{2}}{2 r}|\boldsymbol{\zeta}|^{2}\right]\right|_{t}+O\left(\epsilon^{3 / 2}\right) .
\end{aligned}
$$

Given that, as $\epsilon \rightarrow 0$, a large number of successive tiny time steps will occur before the system appreciably changes position, the positive $|\zeta|^{2}$ term can be replaced by its statistical average $(4.26)$ :

$$
r(t+\epsilon) \approx\left[r-\epsilon V^{\prime}(r)+\epsilon \hat{\mathbf{r}} \cdot \boldsymbol{\zeta}+\frac{\epsilon T}{r}\right](t) .
$$

The distribution of $\hat{\mathbf{r}} \cdot \boldsymbol{\zeta}$ does not care about the direction of $\hat{\mathbf{r}}$, and (4.31) can be rewritten as

$$
r(t+\epsilon) \approx r(t)-\epsilon V_{\mathrm{eff}}^{\prime}(r(t))+\epsilon \zeta_{r}(t),
$$

where $\zeta_{r}$ is uncorrelated white noise and

$$
V_{\text {eff }}(r)=V(r)-T \ln r .
$$

The continuum projected equations that are truly equivalent to the unprojected one are therefore (4.17) or (4.22) with $V$ replaced by $V_{\text {eff. }}$. The addition of the $\ln r$ term in (4.33) now provides a "centrifugal potential" which prevents the one-dimensional system (4.22) from passing through $r=0$.

\section{Equilibrium distributions}

The exact form of the "centrifugal" correction was really determined from the very start. As we shall briefly review in section IVC4, the equilibrium distribution in $\mathbf{r}$ generated by the unprojected equation (4.22) is proportional to $\exp (-V / T)$. That means that the probability distribution for the radial variable $r$ must be proportional to

$$
2 \pi r \exp (-V / T) \propto \exp \left(-V_{\text {eff }} / T\right)
$$

since the $2 \pi r$ is the volume of the symmetry orbit. But $\exp \left(-V_{\text {eff }} / T\right)$ is precisely the equilibrium distribution generated by the projected equation (4.22) if $V$ is replaced by $V_{\text {eff }}$.

In the gauge theory case, there will be no analog to the one-dimensional radial equation (4.22), and so it is worthwhile to understand how the equilibrium distribution could have been deduced directly from the two-dimensional projected equation (4.17). This requires deriving the Fokker-Planck equation that is associated with a given Langevin equation and which describes the time evolution of probability distributions $\mathcal{P}(\mathbf{r})$. (This will be discussed explicitly in the gauge theory case below.) For the naive projected equation (4.17), one finds that

$$
\mathcal{P}(\mathbf{r}) \propto \frac{\exp (-V / T)}{2 \pi r}
$$

is a (two-dimensional) equilibrium solution. But if we correct the naive equation by replacing $V \rightarrow V_{\text {eff }}$, then we indeed recover the unprojected result 


$$
\mathcal{P}(\mathbf{r}) \propto \frac{\exp \left(-V_{\text {eff }} / T\right)}{2 \pi r}=\exp (-V / T)
$$

as the equilibrium distribution in $\mathbf{r}$. Th [Do not confuse the two-dimensional distribution (4.36) for $\mathbf{r}$ with the one-dimensional radial distribution (4.34) for $r$. Both describe the same equilibrium ensemble.]

\section{Gauge Theory}

\section{Time discretization ambiguities}

The transverse-projected version (4.4) of the soft effective theory is a Langevin equation of the form

$$
\begin{gathered}
\frac{d}{d t} q_{i}=-\partial_{i} V(\mathbf{q})+e_{i a}(\mathbf{q}) \zeta_{a}, \\
\left\langle\zeta_{a}(t) \zeta_{b}\left(t^{\prime}\right)\right\rangle=2 \mathcal{T} \delta_{a b} \delta\left(t-t^{\prime}\right),
\end{gathered}
$$

where, for the moment, we are using notation natural for a system with a finite number of degrees of freedom. In the field theory case, the dynamical variables $\mathbf{q}$ are the values of the gauge fields at different points in space and $\partial_{i}$ becomes a functional derivative $\delta / \delta A$. The functions $e_{i a}(\mathbf{q})$ characterize the coupling of the noise to the dynamical variables $\mathbf{q}$; for the gauge theory this is the transverse projection operator $P_{\mathrm{T}}$ (which depends on the gauge field A).

To define exactly what is meant by this equation, imagine discretizing time into very small time steps of size $\epsilon .0$. the coupling $e_{i a}(\mathbf{q})$ to the noise has non-trivial dependence of $\mathbf{q}$, because of the ambiguity, discussed earlier, of when to evaluate q. In the discretized equation,

$$
\begin{gathered}
\frac{q_{i}(t+\epsilon)-q_{i}(t)}{\epsilon}=\left[-\partial_{i} V+e_{i a} \zeta_{a}\right]_{t+\alpha \epsilon}, \\
\left\langle\zeta_{a}(t) \zeta_{b}\left(t^{\prime}\right)\right\rangle=2 \mathcal{T} \epsilon^{-1} \delta_{a b} \delta_{t t^{\prime}},
\end{gathered}
$$

\footnotetext{
${ }^{14}$ Because the projected equation conserves $\theta$, there is a family of two-dimensional equilibrium solutions to the projected toy model equation: namely, the rotationally invariant distribution (4.35) can be multiplied by an arbitrary angular distribution $f(\theta)$. This non-uniqueness is, of course, irrelevant if one is only interested in rotationally invariant observables. The appearance of an arbitrary function of $\theta$ in the general equilibrium distribution is a reflection of the non-ergodicity of the projected two-dimensional evolution equation. As discussed in Appendix B, for the transverse-projected gauge theory there does not appear to be any analog of a conserved gauge-orbit coordinate $\theta$ and, so far as we know, the transverse-projected gauge theory remains ergodic.

15 The following discussion roughly follows the presentation in sections 4.7 and 4.8 of ref. [12], although our normalizations are slightly different.
} 
this ambiguity appears as dependence on a parameter $\alpha$ which controls the time at which the right-hand side is evaluated. For example, $\alpha=0$ corresponds to a forward time derivative and is known as the Itô convention, $\alpha=\frac{1}{2}$ to the symmetric derivative, known as Stratonovich convention, and $\alpha=1$ to a backward time derivative. The precise meaning of evaluation at time $t+\alpha \epsilon$ is to expand in $\alpha \epsilon$. Keeping in mind that the amplitude of the noise $\zeta$ is $\epsilon^{-1 / 2}$, and using the equation of motion itself, the terms in the expansion which are non-negligible when $\epsilon \rightarrow 0$ are

$$
\frac{q_{i}(t+\epsilon)-q_{i}(t)}{\epsilon}=\left[-\partial_{i} V+e_{i a} \zeta_{a}+\alpha \epsilon\left(\partial_{j} e_{i a}\right) e_{j b} \zeta_{a} \zeta_{b}\right]_{t}
$$

The product $\zeta_{a} \zeta_{b}$ may be replaced by its expectation, giving the final discretized equation

$$
\frac{q_{i}(t+\epsilon)-q_{i}(t)}{\epsilon}=\left[-\partial_{i} V+2 \alpha \mathcal{T}\left(\partial_{j} e_{i a}\right) e_{j a}+e_{i a} \zeta_{a}\right]_{t}
$$

The term proportional to $\alpha$ is a convention-dependent "drift" force. The naive continuoustime formulation (4.37) does not, in general, uniquely specify the dynamics.

\section{Vanishing ambiguity}

We shall now show that the ambiguity vanishes for the transverse-only noise equation of (4.4). This implies that there is no real issue of convention dependence for this application.

We work in continuous space (rather than working on a spatial lattice, which would be more relevant to numerical simulations but also more complicated). The degrees of freedom in the gauge theory case are labeled by coordinates $X=(\mathbf{x}, i, a)$, where $i$ is a vector index and $a$ an adjoint color index. It will be convenient to introduce combined labels for several different choices of coordinates:

$$
X=(\mathbf{x}, i, a), \quad Y=(\mathbf{y}, j, b), \quad Z=(\mathbf{z}, k, c) .
$$

The noise coupling $e_{i a}$ introduced above becomes the (matrix elements of the) transverse projection operator

$$
P_{X Y}=\delta^{i j} \delta^{a b} \delta(\mathbf{x}-\mathbf{y})-\left(D^{i} D^{-2} D^{j}\right)_{\mathbf{x y}}^{a b} .
$$

This operator is symmetric in $X$ and $Y$, and the drift force discussed earlier is proportional to

$$
P_{X Z} \frac{\delta}{\delta A_{X}} P_{Z Y} .
$$

When taking the variation of $P_{Z Y}$, the variation must act on the left-most covariant derivative in (4.43), since otherwise that derivative will annihilate against the $P_{X Z}$ factor. One thus obtains

$$
\begin{aligned}
P_{X Z} \frac{\delta}{\delta A_{X}} P_{Z Y} & \propto \int_{\mathbf{z}} P_{X Z} \delta^{i k} T_{c e}^{a} \delta(\mathbf{x}-\mathbf{z})\left(D^{-2} D^{j}\right)_{\mathbf{z y}}^{e b} \\
& \propto d T_{a e}^{a}\left(D^{-2} D^{j}\right)_{\mathbf{x y}}^{e b}-\left(D^{i} D^{-2} D^{i}\right)_{\mathbf{x} \mathbf{x}}^{a c} T_{c e}^{a}\left(D^{-2} D^{j}\right)_{\mathbf{x y}}^{e b}
\end{aligned}
$$


in $d$ spatial dimensions, where no integration over $\mathbf{x}$ is implied. The first term vanishes because the adjoint generators $T_{b c}^{a}$ are anti-symmetric in $(a b c)$ and so $T_{a e}^{a}=0$. The second term vanishes because $\left(D^{i} D^{-2} D^{i}\right)_{\mathbf{x x}}^{a c}$ is symmetric in $(a c)$ and so vanishes when contracted

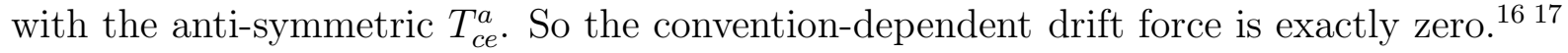

\section{Centrifugal drift}

We now return to the transformation, between Bödeker's effective theory and the transverse-projected theory, in order to derive the gauge theory analog of the centrifugal correction discussed for the toy model in section IVB3. The time-discretized version of Bödeker's effective theory is

$$
\mathbf{A}(t+\epsilon)=\mathbf{A}(t)-\frac{\epsilon}{\sigma}\left(\frac{\delta \mathcal{V}}{\delta \mathbf{A}}(t)-\boldsymbol{\zeta}(t)\right)
$$

where

$$
\mathcal{V}=\frac{1}{2} g^{-2} \int_{\mathbf{x}} B_{i}^{a} B_{i}^{a}=\frac{1}{4} g^{-2} \int_{\mathbf{x}} F_{i j}^{a} F_{i j}^{a}
$$

is the potential energy associated with the magnetic field, the noise correlation is

$$
\left\langle\zeta_{i}^{a}(t, \mathbf{x}) \zeta_{j}^{b}\left(t+n \epsilon, \mathbf{x}^{\prime}\right)\right\rangle=2 g^{2} T \sigma \epsilon^{-1} \delta^{a b} \delta_{i j} \delta_{n 0} \delta\left(\mathbf{x}-\mathbf{x}^{\prime}\right)
$$

and we have chosen to use a forward time difference. We now want to apply (a discrete version of) the gauge transformation $U$ that was introduced in the time-continuum case, (4.10), to eliminate longitudinal motion of A. For simplicity of presentation, we shall focus on one single, time step from $t$ to $t+\epsilon$ and discuss how to transform $\mathbf{A}(t+\epsilon)$ relative to $\mathbf{A}(t)$ in order to eliminate the longitudinal motion introduced during that step. Consider a gauge transformation $U$ which equals the identity at time $t$, but is a non-trivial infinitesimal transformation at time $t+\epsilon$,

$$
U(t, \mathbf{x})=1, \quad U(t+\epsilon, \mathbf{x})=\exp \alpha(\mathbf{x}) .
$$

For the moment, we leave the generator of the transformation, $\alpha$, arbitrary. The gauge transformed field is

\footnotetext{
16 The drift force is also proportional to $\mathcal{T}$ which, in the field theory case, is $T \delta(\mathbf{0})$. If we were only interested in perturbative physics, we could have chosen to work in dimensional regularization, which sets $\delta(\mathbf{0})$ to zero.

${ }^{17}$ In the general case, a sufficient condition for the ambiguity to vanish can be expressed as follows. Suppose the potential $V(\mathbf{q})$ of $(4.37)$ is invariant under some symmetry transformations that have the infinitesimal form $\mathbf{q} \rightarrow \mathbf{q}+\lambda \boldsymbol{\theta}^{\alpha}(\mathbf{q})$, where $\alpha$ indexes the independent symmetry generators (and $\lambda$ is infinitesimal). Define a metric $g^{\alpha \beta}=\boldsymbol{\theta}^{\alpha} \cdot \boldsymbol{\theta}^{\beta}$ on the space of symmetry generators. As in general relativity, let $g_{\alpha \beta}$ denote the (matrix) inverse of the metric. Now suppose that the noise coupling equals the projection operator $e_{i j}=\delta_{i j}-\theta_{i}^{\alpha} g_{\alpha \beta} \theta_{j}^{\beta}$. One can then show that the ambiguity $\left(\partial_{j} e_{i a}\right) e_{j a}$ vanishes if $\partial_{i} \theta_{j}^{\alpha}$ is anti-symmetric under interchange of $i$ and $j$. This anti-symmetry condition is indeed satisfied in both the radial-projected toy model and transverse-projected gauge theory.
} 


$$
\overline{\mathbf{A}} \equiv U(\boldsymbol{\nabla}+A) U^{-1} .
$$

Expanding in powers of the generator $\alpha$ at time $t+\epsilon$, this gives

$$
\overline{\mathbf{A}}(t+\epsilon)=\mathbf{A}(t+\epsilon)-\mathbf{D} \alpha+\frac{1}{2}[\mathbf{D} \alpha, \alpha]+O\left(\alpha^{3}\right),
$$

where $\mathbf{D} \alpha=\boldsymbol{\nabla} \alpha+[\mathbf{A}(t+\epsilon), \alpha]$. Using the equation of motion (4.46) to rewrite $\mathbf{A}(t+\epsilon)$ in terms of $\mathbf{A}(t)$ gives

$$
\overline{\mathbf{A}}(t+\epsilon)=\mathbf{A}(t)-\frac{\epsilon}{\sigma}\left(\frac{\delta \mathcal{V}}{\delta \mathbf{A}}-\boldsymbol{\zeta}\right)-\mathbf{D} \alpha+\frac{\epsilon}{\sigma}\left[\frac{\delta \mathcal{V}}{\delta \mathbf{A}}-\boldsymbol{\zeta}, \alpha\right]+\frac{1}{2}[\mathbf{D} \alpha, \alpha]+O\left(\alpha^{3}, \sqrt{\epsilon} \alpha^{2}\right),
$$

where now all the covariant derivatives involve the gauge field at time $t$. Choosing the infinitesimal generator to equal

$$
\alpha=\frac{\epsilon}{\sigma} D^{-2} \mathbf{D} \cdot \boldsymbol{\zeta}
$$

as implied by our previous discussion [c.f. (4.10)], will cause the $\mathbf{D} \alpha$ term to cancel the longitudinal projection of the noise $\boldsymbol{\zeta}$. Since the noise $\boldsymbol{\zeta}$ is of order $\epsilon^{-1 / 2}$, this means that $\alpha$ is of order $\sqrt{\epsilon}$. We need to keep all terms in (4.52) which are of order $\epsilon$. The term $(\epsilon / \sigma)[\delta \mathcal{V}, \alpha]$ is $O\left(\epsilon^{3 / 2}\right)$ and may be neglected. Consequently,

$$
\overline{\mathbf{A}}(t+\epsilon)=\mathbf{A}(t)+\frac{\epsilon}{\sigma}\left(-\frac{\delta \mathcal{V}}{\delta \mathbf{A}}+P_{\mathrm{T}} \boldsymbol{\zeta}\right)-\frac{\epsilon^{2}}{\sigma^{2}}\left[\boldsymbol{\zeta}-\frac{1}{2} \mathbf{D} D^{-2} \mathbf{D} \cdot \boldsymbol{\zeta}, D^{-2} \mathbf{D} \cdot \boldsymbol{\zeta}\right]+O\left(\epsilon^{3 / 2}\right) .
$$

Once again, we can replace the terms quadratic in noise by their statistical averages, as given by (4.48). After some manipulation, one finds that this yields

$$
\begin{aligned}
\overline{\mathbf{A}}^{a}(t+\epsilon, \mathbf{x}) & =\mathbf{A}^{a}(t, \mathbf{x})+\frac{\epsilon}{\sigma}\left(-\frac{\delta \mathcal{V}}{\delta \mathbf{A}^{a}(\mathbf{x})}+P_{\mathrm{T}} \boldsymbol{\zeta}^{a}(t, \mathbf{x})\right)+\frac{T \epsilon}{\sigma} f^{a b c}\left(\mathbf{D} D^{-2}\right)_{\mathbf{x x}}^{c b}+O\left(\epsilon^{3}\right) \\
& =\mathbf{A}^{a}(t, \mathbf{x})+\frac{\epsilon}{\sigma}\left(-\frac{\delta \mathcal{V}_{\mathrm{eff}}}{\delta \mathbf{A}^{a}(\mathbf{x})}+P_{\mathrm{T}} \boldsymbol{\zeta}^{a}(t, \mathbf{x})\right)+O\left(\epsilon^{3}\right)
\end{aligned}
$$

where the effective potential $\mathcal{V}_{\text {eff }}$ is

$$
\mathcal{V}_{\text {eff }}=\mathcal{V}-\frac{1}{2} T \operatorname{Tr} \ln \left(-D^{2}\right)=\mathcal{V}-T \ln \sqrt{\operatorname{det}\left(-D^{2}\right)}
$$

As shown in Appendix $\mathbb{A}, \sqrt{\operatorname{det}\left(-D^{2}\right)}$ is the volume of the gauge orbit containing a given spatial gauge field. Consequently, this logarithmic correction to the potential is completely analogous to the "centrifugal" potential appearing in the rotationally invariant toy model. The upshot is that the projected equation which is actually equivalent to Bödeker's effective theory differs from the naive projected equation (4.4) by the replacement of $\mathcal{V}$ by $\mathcal{V}_{\text {eff }}$ :

$$
\sigma \frac{d}{d t} \mathbf{A}=-\mathbf{D} \times \mathbf{B}+\frac{1}{2} T \frac{\delta}{\delta \mathbf{A}} \operatorname{Tr} \ln \left(-D^{2}\right)+P_{\mathrm{T}} \boldsymbol{\zeta} .
$$




\section{The equilibrium distribution}

It's interesting to examine what happens if one converts a Langevin equation of the generic form (4.41) into a Fokker-Planck equation for the evolution of the probability distribution $\mathcal{P}(\mathbf{q}, t)$. One finds (see for example [12]) that

$$
\frac{\partial}{\partial t} \mathcal{P}=\partial_{i}\left[\mathcal{T} \partial_{j}\left(e_{i a} e_{j a} \mathcal{P}\right)+\left\{\partial_{i} V-2 \alpha \mathcal{T}\left(\partial_{j} e_{i a}\right) e_{j a}\right\} \mathcal{P}\right]
$$

If $e_{i a}(\mathbf{q})$ were just $\delta_{i a}$, as in Bödeker's equation (4.3), then the equilibrium distribution (the solution to $d \mathcal{P} / d t=0)$ would simply be $\mathcal{P}=\exp (-V / \mathcal{T})$ up to an overall normalization constant.

For the naive radial-projected toy model (4.17) with $e_{i a}=\hat{r}_{i} \hat{r}_{a}$,

$$
\partial_{j}\left(e_{i a} e_{j a}\right)=\frac{\hat{r}_{i}}{r}=\hat{r}_{i} \frac{d}{d r} \ln (2 \pi r),
$$

and $\left(\partial_{j} e_{i a}\right) e_{j a}=0$. This leads to the equilibrium distributions (4.35) quoted in section IVB 4 .

In the gauge theory case, we have seen that the convention-dependent drift force vanishes for the transverse-only noise equation (4.4), but there still remains $e_{i a}$ dependence in the Fokker-Planck equation. Plugging in the transverse projection operator for $e$, one finds

$$
\partial_{j}\left(e_{i a} e_{j a}\right) \rightarrow \frac{\delta}{\delta A_{Y}} P_{X Y}=\frac{1}{2} P_{X Y} \frac{\delta}{\delta A_{Y}} \operatorname{tr} \ln \left(-D^{2}\right),
$$

and, solving for the equilibrium distribution,

$$
\mathcal{P}=\frac{\exp (-\mathcal{V} / T)}{\sqrt{\operatorname{det}\left(-D^{2}\right)}}
$$

up to an overall normalization constant. 18 As mentioned above, $\sqrt{\operatorname{det}\left(-D^{2}\right)}$ just represents the gauge orbit volume, and the above distribution is analogous to the toy model result (4.35). As with the toy model, however, if we examine the transverse theory that is truly equivalent to the unprojected theory, then we should replace $\mathcal{V} \rightarrow \mathcal{V}_{\text {eff }}$ and we recover the correct equilibrium distribution

$$
\mathcal{P}=\frac{\exp \left(-\mathcal{V}_{\text {eff }} / T\right)}{\sqrt{\operatorname{det}\left(-D^{2}\right)}}=\exp (-\mathcal{V} / T)
$$

\footnotetext{
18 In the more general notation of footnote 17, the assumption that $\partial_{i} \theta_{j}^{\alpha}$ is anti-symmetric in $i$ and $j$ leads to $\partial_{j}\left(e_{i a} e_{j a}\right)=\frac{1}{2} e_{i j} \partial_{j} \ln \sqrt{g}$ and $\mathcal{P}=\exp (-V / T) / \sqrt{g}$, where $g$ is the determinant of the inverse metric $g_{\alpha \beta}$.
} 


\section{ACKNOWLEDGMENTS}

We thank Dietrich Bödeker and Guy Moore for useful conversations. We are especially indebted to Deitrich Bödeker for conversations, concerning an earlier version of this manuscript, which inspired us to clarify our understanding of the $\omega \ll k \ll \gamma_{\mathrm{g}}$ limit of the longitudinal sector. This work was supported, in part, by the U.S. Department of Energy under Grant Nos. DE-FG03-96ER40956 and DF-FC02-94ER40818.

\section{APPENDIX A: THE VOLUME OF GAUGE ORBITS}

The natural metric on the space of gauge field vector potentials is

$$
d s^{2}=\int_{\mathbf{x}} \operatorname{tr}\left[d \mathbf{A}^{\dagger} \cdot d \mathbf{A}\right]
$$

This is the unique metric (up to an overall multiplicative constant) which is invariant under both gauge transformations and spacetime symmetries. The gauge orbit passing through a particular gauge configuration $\mathbf{A}$ consists of all gauge transforms of $\mathbf{A}$. Within a neighborhood of $\mathbf{A}$, configurations on the gauge orbit may be parameterized as

$$
\mathbf{A}^{\Lambda} \equiv e^{-\Lambda} \mathbf{D} e^{\Lambda}
$$

where $\Lambda$ is an arbitrary generator of the gauge group (i.e., $\Lambda(\mathbf{x}) \equiv \Lambda^{a}(\mathbf{x}) T^{a}$ is a Lie-algebra valued function of $\mathbf{x}$ ), and $\mathbf{D}=\boldsymbol{\nabla}+\mathbf{A}$ is the covariant derivative with gauge field $\mathbf{A}$. Since $\mathbf{A}^{\Lambda}-\mathbf{A} \sim \mathbf{D} \Lambda$, the induced metric on the gauge orbit, evaluated at $\mathbf{A}$, is just

$$
\left.d s^{2}\right|_{\text {orbit }}=\int_{\mathbf{x}} \operatorname{tr}\left[(\mathbf{D} \delta \Lambda)^{\dagger} \cdot(\mathbf{D} \delta \Lambda)\right]=\int_{\mathbf{x}} \operatorname{tr}\left[\delta \Lambda^{\dagger}\left(-D^{2}\right) \delta \Lambda\right]
$$

Consequently the induced volume element on the orbit, evaluated at $\mathbf{A}$, is

$$
d v=\sqrt{\operatorname{det}\left(-D^{2}\right)} d \Lambda
$$

where $d \Lambda \equiv \prod_{\mathbf{x}, a} d \Lambda^{a}(\mathbf{x})$ denotes the flat measure on the gauge algebra. But the gaugeinvariant Haar measure on the gauge group is just $d \Lambda$ when evaluated at the identity. And, because the functional determinant $\operatorname{det}\left(-D^{2}\right)$ is gauge invariant, it is constant over the

gauge orbit. So, globally, the volume element $d v$ equals $\sqrt{\operatorname{det}\left(-D^{2}\right)}$ times the Haar measure on the gauge group. Hence,

$$
\frac{\text { orbit volume }}{\text { gauge group volume }}=\left[\operatorname{det}\left(-D^{2}\right)\right]^{1 / 2},
$$

and so $\sqrt{\operatorname{det}\left(-D^{2}\right)}$ is the gauge orbit volume up to an overall A-independent normalization factor. 


\section{APPENDIX B: NO GAUGE THEORY ANALOG TO TOY MODEL $\theta$}

Return, for a moment, to the toy model described in Sec. IVB. The projected equation (4.17) has two obvious properties. First, the particle always moves in a direction perpendicular to the gauge orbits $r=$ const. Second, moving according to this equation, the particle cannot reach any point in the configuration space, but instead remains confined to a slice in a configuration space where $\theta$ is a constant. In particular, starting from a point $(r, \theta)$, one cannot reach any point that is gauge equivalent to it, except the original point. In other words, if one fixes the gauge $\theta=\theta_{0}$, with $\theta_{0}$ some constant, then this gauge-fixing condition remains satisfied throughout the random walk.

Now consider the gauge theory. Eq. (4.4) describes a motion in the space of field configurations that is analogous to that described by the projected equation (4.17) in the toy model. In terms of the natural metric (A1), on the space of gauge configurations, one can easily check that the motion is always along directions perpendicular to deformations generated by gauge transformations (This is equivalent to satisfying the condition $\mathbf{D} \cdot \dot{\mathbf{A}}=0$.) The question we want to ask is whether the second property of our toy model still holds, i.e., is the motion confined to a slice in configuration space? It might be surprising that the answer to this question is negative, and one can travel throughout the whole configuration space even when restricted to trajectories whose tangents, at every point, are perpendicular to the intersecting gauge orbit. This negative answer is perhaps less surprising if one notices that there is no obvious gauge-fixing condition similar to $\theta=\theta_{0}$ that is conserved during the transverse-projected random walk (4.4). Thus, in the gauge theory, there is no equivalence of the parameter $\theta$.

This can be seen most directly by the explicit construction of a trajectory that remains perpendicular to gauge transformations at all times, but nevertheless connects two distinct points on the same gauge orbit. The trajectory we are going to construct starts at $\mathbf{A}=0$ and remains small all the time, so we can use perturbation theory. Let us denote the small parameter by $\epsilon$. Consider first the following trajectory,

$$
A_{i}(t, \mathbf{x})= \begin{cases}t C_{i}(\mathbf{x}), & 0<t<\epsilon \\ \epsilon C_{i}(\mathbf{x})+(t-\epsilon) D_{i}(\mathbf{x}), & \epsilon<t<2 \epsilon \\ (3 \epsilon-t) C_{i}(\mathbf{x})+\epsilon D_{i}(\mathbf{x}), & 2 \epsilon<t<3 \epsilon \\ (4 \epsilon-t) D_{i}(\mathbf{x}), & 3 \epsilon<t<4 \epsilon\end{cases}
$$

Provided that $C_{i}$ and $D_{i}$ are transverse, $\partial_{i} C_{i}=\partial_{i} D_{i}=0$, it is trivial to check that $\mathbf{D} \cdot \dot{\mathbf{A}}=0$ to leading order in $\epsilon$. This means the trajectory is everywhere perpendicular (to within $\left.O\left(\epsilon^{2}\right)\right)$ to the gauge orbits it passes through.

This trajectory may seem uninteresting, since it is a closed loop that starts at $\mathbf{A}=0$ and ends at the same point. The interest arises when we go to next-to-leading order in $\epsilon$. At next-to-leading order, the trajectory (B1) does not satisfy the condition $\mathbf{D} \cdot \dot{\mathbf{A}}=0$. For example, when $\epsilon<t<2 \epsilon, \mathbf{D} \cdot \dot{\mathbf{A}}=\epsilon\left[C_{i}, D_{i}\right]$. To correct for this deviation, we need to modify $A_{i}$ in the following way:

$$
A_{i}(t, \mathbf{x})=\epsilon C_{i}(\mathbf{x})+(t-\epsilon) D_{i}(\mathbf{x})+(t-\epsilon) \alpha_{i}(\mathbf{x}), \quad \epsilon<t<2 \epsilon,
$$

where $\alpha_{i}=O(\epsilon)$, so the term involving $\alpha_{i}$ is of higher order than the other terms. Eq. (B2) satisfies the condition $\mathbf{D} \cdot \dot{\mathbf{A}}=0$ if one places the following constraint on $\alpha_{i}$ : 


$$
\partial_{i} \dot{\alpha}_{i}+\epsilon\left[C_{i}, D_{i}\right]=0
$$

This condition can be satisfied by choosing

$$
\dot{\alpha}_{i}=-\epsilon \partial_{i} \nabla^{-2}\left[C_{j}, D_{j}\right]
$$

In this manner, one can modify the whole trajectory (B1) so that the condition $\mathbf{D} \cdot \dot{\mathbf{A}}=0$ is satisfied through next-to-leading order. The result is

$$
A_{i}(t, \mathbf{x})= \begin{cases}t C_{i}, & 0<t<\epsilon \\ \epsilon C_{i}+(t-\epsilon) D_{i}-\epsilon(t-\epsilon) \partial_{i} \boldsymbol{\nabla}^{-2}\left[C_{j}, D_{j}\right], & \epsilon<t<2 \epsilon \\ (3 \epsilon-t) C_{i}+\epsilon D_{i}-\left(\epsilon^{2}+\epsilon(t-2 \epsilon)\right) \partial_{i} \boldsymbol{\nabla}^{-2}\left[C_{j}, D_{j}\right], & 2 \epsilon<t<3 \epsilon \\ (4 \epsilon-t) D_{i}-2 \epsilon^{2} \partial_{i} \boldsymbol{\nabla}^{-2}\left[C_{j}, D_{j}\right], & 3 \epsilon<t<4 \epsilon\end{cases}
$$

One sees that the trajectory now starts at $A_{i}=0$ at $t=0$ and runs to $A_{i}=$ $-2 \epsilon^{2} \partial_{i} \boldsymbol{\nabla}^{-2}\left[C_{j}, D_{j}\right]$ at $t=4 \epsilon$. [Including still higher-order corrections would only change this by $O\left(\epsilon^{3}\right)$.] At the end point, $\mathbf{A}$ is a pure gauge, and, for a general choice of $C_{i}$ and $D_{i}$, nonzero. Therefore, this trajectory presents a simple example of how, following a transverse projected trajectory, one can go from one field configuration to a field configuration that is gauge equivalent to it. From this result one may show (provided the gauge group is semi-simple) that any field configuration in the vicinity of $\mathbf{A}=0$ is accessible to the transverse-projected random walk (4.4). Hence, there can be no analog of the toy model "slice parameter" $\theta$ for transverse-projected dynamics in non-Abelian gauge theories. 


\section{REFERENCES}

[1] P. Arnold, D. Son, and L. Yaffe, hep-ph/9609481, Phys. Rev. D55, 6264 (1997).

[2] R. Pisarski, Phys. Rev. Lett. 63, 1129 (1989); Phys. Rev. D47, 5589 (1993).

[3] V. Lebedev and A. Smilga, Physica A181, 187 (1992).

[4] J. Blaizot and E. Iancu, Phys. Rev. Lett. 76, 3080 (1996); Phys. Rev. D55, 973 (1997); D56, 7877.

[5] A. Selikhov and M. Gyulassy, nucl-ph/9307007, Phys. Lett. B316, 373 (1993).

[6] P. Arnold, D. Son, and L. Yaffe, hep-ph/9810216, University of Washington preprint UW/PT 98-10.

[7] D. Bödeker, hep-ph/9810430, Phys. Lett. B426, 351 (1998).

[8] A. Selikhov and M. Gyulassy, nucl-ph/9307007, Phys. Lett. B316, 373 (1993).

[9] G. Moore, hep-ph/9810313, McGill preprint MCGILL-98-28.

[10] See, for example, J. Kapusta, Finite Temperature Field Theory, (Cambridge University Press, 1989).

[11] P. Arnold, D. Son, and L. Yaffe, hep-ph/9609481, Phys. Rev. D55, 6264 (1997).

[12] J. Zinn-Justin, Quantum Field Theory and Critical Phenomena, 2nd edition (Oxford University Press, 1993). 